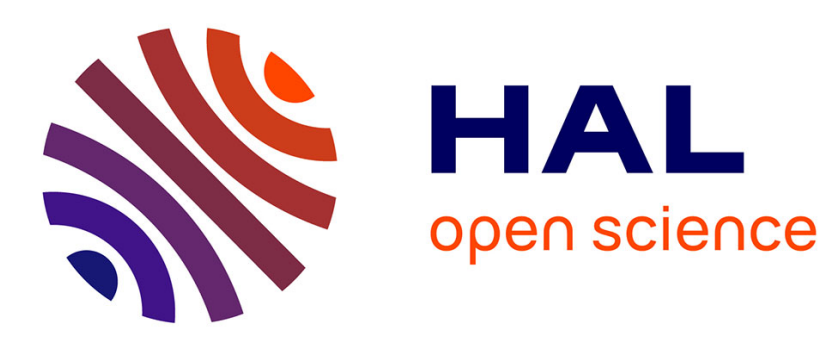

\title{
Bayesian Dynamic Finite-Fault Inversion: 1. Method and Synthetic Test
}

F. Gallovič, L. Valentová, J.-p. Ampuero, A.-a. Gabriel

\section{To cite this version:}

F. Gallovič, L. Valentová, J.-p. Ampuero, A.-a. Gabriel. Bayesian Dynamic Finite-Fault Inversion: 1. Method and Synthetic Test. Journal of Geophysical Research: Solid Earth, 2019, 124 (7), pp.69496969. 10.1029/2019JB017510 . hal-02372788

\section{HAL Id: hal-02372788 \\ https://hal.science/hal-02372788}

Submitted on 16 Sep 2021

HAL is a multi-disciplinary open access archive for the deposit and dissemination of scientific research documents, whether they are published or not. The documents may come from teaching and research institutions in France or abroad, or from public or private research centers.
L'archive ouverte pluridisciplinaire HAL, est destinée au dépôt et à la diffusion de documents scientifiques de niveau recherche, publiés ou non, émanant des établissements d'enseignement et de recherche français ou étrangers, des laboratoires publics ou privés.

$$
\text { Copyright }
$$




\section{RESEARCH ARTICLE 10.1029/2019JB017510}

This article is a companion to Gallovic et al. (2019), https://doi.org/10.1029/ 2019JB017512.

Key Points:

- A novel Bayesian dynamic earthquake source inversion with spatially variable prestress and slip-weakening friction parameters is introduced

- Application to community benchmark test shows that the dynamic inversion reduces the uncertainty of kinematic parameters

- Highly efficient dynamic rupture solvers are required for including physics-based constraints in slip inversions

Correspondence to:

F. Gallovič,

gallovic@karel.troja.mff.cuni.cz

Citation:

Gallovič, F., Valentová, L'., Ampuero, J.P., \& Gabriel, A.-A. (2019). Bayesian dynamic finite-fault inversion: 1 . Method and synthetic test. Journal of Geophysical Research: Solid Earth, 124, 6949-6969. https://doi.org/10.1029/ 2019JB017510

Received 7 FEB 2019

Accepted 19 JUN 2019

Accepted article online 26 JUN 2019

Published online 22 JUL 2019

(c)2019. American Geophysical Union. All Rights Reserved.

\section{Bayesian Dynamic Finite-Fault Inversion: 1. Method and Synthetic Test}

\author{
F. Gallovič ${ }^{1}\left[\right.$, L. Valentová ${ }^{1}$, J.-P. Ampuero ${ }^{2}$ iD, and A.-A. Gabriel ${ }^{3}$ (iD \\ ${ }^{1}$ Department of Geophysics, Faculty of Mathematics and Physics, Charles University, Praha, Czech Republic, ${ }^{2}$ Université \\ Cote d'Azur, IRD, CNRS, Observatoire de la Cote d'Azur, Sophia Antipolis, Cedex, France, ${ }^{3}$ Department of Earth and \\ Environmental Sciences, Geophysics, Ludwig-Maximilians-Universität München, München, Germany
}

\begin{abstract}
Dynamic earthquake source inversions aim to determine the spatial distribution of initial stress and friction parameters leading to dynamic rupture models that reproduce observed ground motion data. Such inversions are challenging, particularly due to their high computational burden; thus, so far, only few attempts have been made. Using a highly efficient rupture simulation code, we introduce a novel method to generate a representative sample of acceptable dynamic models from which dynamic source parameters and their uncertainties can be assessed. The method assumes a linear slip-weakening friction law and spatially variable prestress, strength, and characteristic slip-weakening distance along the fault. The inverse problem is formulated in a Bayesian framework, and the posterior probability density function is sampled using the Parallel Tempering Monte Carlo algorithm. The forward solver combines a 3-D finite difference code for dynamic rupture simulation on a simplified geometry to compute slip rates and precalculated Green's functions to compute ground motions. We demonstrate the performance of the proposed method on a community benchmark test for source inversion. We find that the dynamic parameters are resolved well within the uncertainty, especially in areas of large slip. The overall relative uncertainty of the dynamic parameters is rather large, reaching $50 \%$ of the averaged values. In contrast, the kinematic rupture parameters (rupture times, rise times, and slip values), also well resolved, have relatively lower uncertainties of $\sim 10 \%$. We conclude that incorporating physics-based constraints, such as an adequate friction law, may serve also as an effective constraint on the rupture kinematics in finite-fault inversions.
\end{abstract}

\section{Introduction}

Finite-fault earthquake source inversions are typically based on a kinematic description of the rupture process. The source at each point on the fault is parameterized in terms of a slip rate function, either with prescribed shape in nonlinear inversions (Cirella et al., 2009; Ji et al., 2002; Monelli \& Mai, 2008; Piatanesi et al., 2007) or as a linear combination of triangular basis functions in linear inversions (Delouis et al., 2002; Fan et al., 2014; Frankel \& Wennerberg, 1989; Gallovič et al., 2009; Gallovič et al., 2015; Gallovič \& Zahradník, 2011; Hartzell \& Heaton, 1983; Olson \& Anderson, 1988; Sekiguchi et al., 2002; Song \& Dalguer, 2017). The result of kinematic source inversion is a model (or set of models) describing the space-time distribution of slip rate along the fault, from which the rupture propagation can be interpreted. It is generally accepted that such solutions are strongly nonunique due to the ill-conditioning of the kinematic inverse problem (e.g., Clévédé et al., 2004; Gallovič \& Zahradník, 2011; Hartzell et al., 2007; Gallovič \& Ampuero, 2015; Mai et al., 2016; Shao \& Ji, 2012; Zahradník \& Gallovič, 2010). Moreover, kinematic models provide only limited access to the physical properties that control rupture nucleation, propagation, and arrest, such as the state of stress on the fault and its friction properties. Indeed, the inference of dynamic parameters from kinematic ones is nontrivial and depends on assumptions made in the kinematic inversion on, for example, the shape of the slip rates or model smoothness (Tinti et al., 2009).

The dynamic description of earthquake sources is mostly based on small-scale laboratory experiments and theoretical considerations. The appropriate form of the constitutive law that describes the relationship between the fault stress and slip is a topic of intense research. Widely applied empirical friction laws, such as the slip-weakening and rate-and-state friction laws, are derived from small-scale laboratory experiments (Brace \& Byerlee, 1966; Ruina, 1983; Niemeijer et al., 2010; Ohnaka, 2013; etc.), and a proper scaling of the frictional parameters to larger magnitude events is a subject of intense research (e.g., Viesca \& Garagash, 
2015). Therefore, it is desirable to infer dynamic rupture parameters of an assumed friction law directly from the observed data. Such inferred model parameters have the potential to shed new light on earthquake physics.

There are several viable approaches to build physics-based earthquake source models consistent with nonlinear rupture dynamics. The most general approach, so-called dynamic source inversion, aims to optimize the spatial distribution of initial stress and frictional parameters along the fault such that the resulting dynamic rupture produces ground motions that fit observed data. Such inversions are particularly challenging due to their high computational burden. So far, only few attempts have been made (Corish et al., 2007; Fukuyama \& Mikumo, 1993; Peyrat \& Olsen, 2004). In particular, Corish et al. (2007) and Peyrat and Olsen (2004) considered constant parameters of the friction law and piecewise constant spatial distributions of prestress. To improve the computational efficiency, some have considered parsimonious parameterizations, such as models consisting of one or two patches with dynamic parameters having uniform or Gaussian spatial distributions (e.g., Ruiz \& Madariaga, 2011; Twardzik et al., 2014). A more common approach is to build a dynamic model from previously inferred kinematic source models (Ide \& Takeo, 1996; Olsen et al., 1997; Peyrat et al., 2001; Tanırcan et al., 2017; Ulrich et al., 2019; Weng \& Yang, 2018) or to analyze slip, slip rate, and stresses computed from kinematic source models to constrain the governing friction law (Bouchon, 1997; Burjánek \& Zahradník, 2007; Goto et al., 2012; Guatteri et al., 2001; Zhang et al., 2003; etc.). Information gained is then used to constrain a detailed dynamic model of the studied earthquake. However, such models can be biased by the choice of inversion constraints, for example, smoothing in the kinematic inversions (Tinti et al., 2009). Therefore, we opt to use the dynamic source inversion with general description of dynamic source parameters, but capitalizing on recent computational advances.

In general, dynamic inversion is a computationally demanding task for two reasons. First, each forward problem, a rupture simulation for a given set of dynamic source parameters, is a computationally intensive task. Second, due to the strongly nonlinear relation between the observed data and the dynamic parameters, the model space has to be explored with a general technique, such as a Monte Carlo approach, which requires a large number of (expensive) forward calculations. In this paper we propose ways to tackle both of these problems in very efficient ways to make the dynamic inversion feasible.

Estimates of the uncertainties associated with the earthquake source inversion results are essential to guarantee the reliability and assess the limits of the interpretation of the inferred models. The Monte Carlo approach in the Bayesian framework is suitable to treat a highly nonlinear forward problem such as dynamic rupture. In a probabilistic sense it combines prior information on the model parameters and constraints provided by the observed data, yielding a posterior probability density function (PDF) that carries information on both the optimal model and its uncertainty. The posterior PDF is typically explored by sampling utilizing a Markov chain Monte Carlo (MCMC) technique. The Bayesian approach has been already applied to kinematic slip inversions (Duputel et al., 2015; Monelli \& Mai, 2008), yet to the best of our knowledge, it has not been utilized in dynamic source inversion studies as done here. Corish et al. (2007), Ruiz and Madariaga (2013), and Peyrat and Olsen (2004) used the neighborhood algorithm to perform model search in the parameter space in their dynamic inversions.

In the present paper we introduce a finite-fault dynamic source inversion approach including evaluation of parameter uncertainties by means of Bayesian inference. We have developed a code (fd3d_pt) for this purpose, consisting of two major components: (i) a direct solver, which, for a given distribution of the dynamic source parameters along the fault (initial stress, strength, and characteristic slip-weakening distance), solves the rupture propagation and predicts the data that would have been observed by the receivers and (ii) a Monte Carlo Parallel Tempering module that samples the posterior PDF in the model space to provide a model that best fits the observed data and the model's uncertainty. After describing the method in section 2, in section 3 we demonstrate its performance on the community benchmark Inv1 of the source inversion validation (SIV) project (Mai et al., 2016). We describe how the inversion is started from a model estimated from preliminary kinematic inversion for more efficient Monte Carlo sampling. In section 4 we summarize lessons learned from the synthetic test. We discuss the uncertainty of the dynamic model parameters in relation with that of the kinematic parameters, pointing out that dynamic source inversions can be viewed as kinematic inversions constrained by the assumed friction law. The remaining question is how well can a dynamic model with finely tuned parameters fit data of a real, well-observed event. This is 
A)

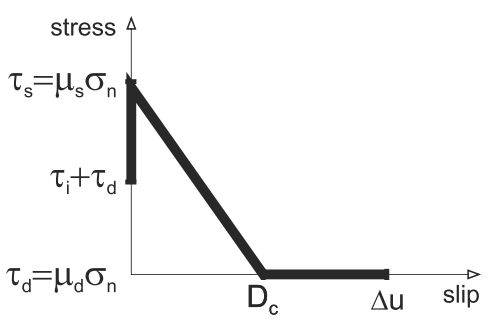

C)

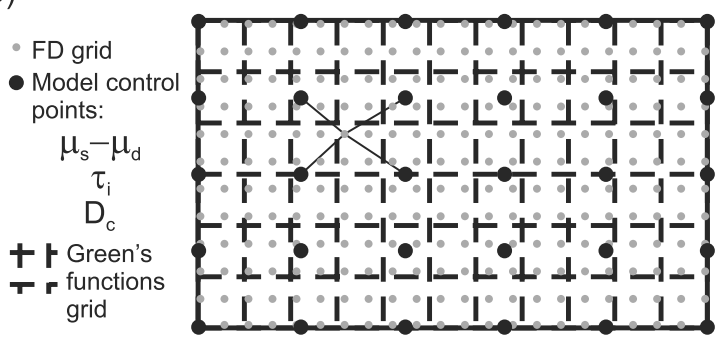

Figure 1. Rupture model and its parameterization. (a) Linear slip-weakening friction law. Parameters subject to inversion are prestress (initial stress) $\tau_{i}$, difference between static and dynamic friction coefficients $\left(\mu_{s}-\mu_{d}\right)$, and characteristic slip-weakening distance $D_{c}$. The frictional coefficient difference is converted to breakdown stress drop $\left(\tau_{s}-\tau_{d}\right)$ by multiplication with a prescribed normal stress depth profile (b). (c) Illustration of three grids considered in the calculations. Model parameters are defined on the coarsest grid of control points. The values are bilinearly interpolated onto the densest finite difference (FD) grid that is used by the FD3D dynamic rupture simulator. Slip rates calculated by FD3D are averaged spatially onto the coarser Green's functions grid, for which Green's functions are precalculated and stored. Waveforms are calculated by convolving the individual averaged slip rates with the respective Green's functions and summed up following the representation theorem. For the actual grid parameters and normal stress profile considered in the present study see Table 1. demonstrated in our companion paper (Gallovič et al., 2019) by performing a dynamic inversion of the $M_{w} 6.22016$ Amatrice, Central Italy, earthquake.

\section{Method}

The structure of this section is as follows. After introducing the Bayesian formulation of our dynamic inversion in section 2.1, we detail the choice of our model parameterization in section 2.2. Section 2.3 describes the highly efficient dynamic rupture code used to solve the forward problem. In section 2.4 we describe the Parallel Tempering Monte Carlo approach that is employed to sample the posterior PDF.

\subsection{Bayesian Formulation of the Inverse Problem}

In the Bayesian framework (Tarantola, 2005; Tarantola \& Valette, 1982; etc.), probabilistic information on model parameters $\boldsymbol{m}$ and on the fit between modeled and observed data $\boldsymbol{d}$, namely, their respective PDFs, is combined to get a posterior PDF of the model. Denoting the prior PDF $p(\boldsymbol{m})$ and the PDF of data $\boldsymbol{d}$ given the model parameters as $p(\boldsymbol{d} \mid \boldsymbol{m})$, the posterior PDF $p(\boldsymbol{m} \mid \boldsymbol{d})$, which is the solution of the inverse problem, reads

$$
p(\boldsymbol{m} \mid \boldsymbol{d})=\frac{p(\boldsymbol{m}) p(\boldsymbol{d} \mid \boldsymbol{m})}{p(\boldsymbol{d})},
$$

where the Bayesian evidence $p(\boldsymbol{d})$ serves as a normalization constant.

The prior PDF $p(\boldsymbol{m})$ is specified (in the next section) by constraints on the plausible values of the model parameters, assumed or derived independently of the current data measurements. The PDF of the data (observed waveforms) given a model, $p(\boldsymbol{d} \mid \boldsymbol{m})$, is assumed to be Gaussian with diagonal covariance function:

$$
p(\boldsymbol{d} \mid \boldsymbol{m})=c_{1} \exp \left(-\frac{1}{2} \sum_{i=1}^{N} \frac{\left\|\boldsymbol{s}_{i}(\boldsymbol{m})-\boldsymbol{d}_{i}\right\|^{2}}{\sigma_{i}^{2}}\right) .
$$

Here $\boldsymbol{d}_{i}$ and $\boldsymbol{s}_{i}(\boldsymbol{m})$ are observed data and synthetics at station $i$, respectively, and $N$ is the number of stations. The symbol $\|\cdot\|$ denotes the $\mathrm{L} 2 \mathrm{norm}$, and $\sigma_{i}$ are the assumed standard deviations representing the combined uncertainty of the modeling and data errors.

\subsection{Model Parameterization}

We assume that the rupture nucleation, propagation, and arrest are governed by the linear slip-weakening friction law (Figure 1a) with spatially inhomogeneous parameters and by spatially variable prestress (also called initial stress). Slip at a given point starts when the stress reaches the local strength $\tau_{s}$ given by the static coefficient of friction $\mu_{s}$ multiplied by the normal stress $\sigma$. The normal stress is the sum of a prescribed static depth profile (Figure $1 \mathrm{~b}$ and Table 1 ) and a dynamic time-dependent component due to seismic radiation. Upon slip, the friction coefficient decays linearly with slip $D$ until the characteristic slip-weakening distance $D_{c}$ is reached. With further slip the friction coefficient remains constant and equal to the dynamic friction coefficient $\mu_{d}$.

We assume that the absolute level of shear stress and strength does not play a significant role in rupture propagation. Thus, we arbitrarily set the reference level of shear stress and strength equal to the dynamic strength $\tau_{d}=\mu_{d} \sigma$. This implies that we consider shear stresses relative to $\mu_{d} \sigma$, we ignore the effect of timedependent normal stress changes on dynamic strength, and we ignore rake rotations caused by shear stress changes comparable to the dynamic strength. The parameters subject to the inversion are the relative prestress $\tau_{i}=\tau_{0}-\tau_{d}$, the characteristic slip-weakening distance $D_{c}$, and the difference between the static and 
Table 1

Model and Computational Parameters Considered for the Source Inversion Validation Inv1 Tests

\begin{tabular}{lc}
\hline Parameter & Value \\
\hline General & \\
Fault mechanism & Strike: $90^{\circ}$, dip: $80^{\circ}$, rake: $180^{\circ}$ \\
Fault dimensions & Length $36 \mathrm{~km}$, width $20 \mathrm{~km}$ \\
Fault top depth & $0 \mathrm{~km}$ \\
Normal stress depth dependence & $16.2 \mathrm{MPa} / \mathrm{km}$ \\
Data error & $0.1 \mathrm{~m}$ \\
FD3D & \\
Spatial discretization & $0.1 \mathrm{~km}$ \\
FD half-domain size (along strike $\times$ normal $\times$ along dip) & $360 \times 100 \times 200$ \\
Duration of slip-rate functions & $10 \mathrm{~s}$ \\
Time step & $0.002 \mathrm{~s}$ \\
Green's functions & \\
Spatial fault discretization & $1 \times 1 \mathrm{~km}$ \\
Time sampling & $0.2 \mathrm{~s}$ \\
Waveform frequency range (displacements) & $0.05-0.5 \mathrm{~Hz}$ \\
Model parameterization and priors & \\
Control point grid (along strike $\times$ along dip) & $13 \times 9$ \\
Prestress $\left(\tau_{i}\right)$ prior & $0-200 \mathrm{MPa}$ \\
Static - dynamic friction coefficient $\left(\mu_{s}-\mu_{d}\right)$ prior & $0-1.1$ \\
Characteristic slip-weakening distance $\left(D_{c}\right)$ prior & $0.15-5.00 \mathrm{~m}$ \\
Nucleation area prior (along-strike, updip, and radius) & $27 \mathrm{~km}, 6 \mathrm{~km}, 5 \mathrm{~km}$
\end{tabular}

dynamic friction coefficients $\mu_{s}-\mu_{d}$, also called friction drop. Note that the latter can be converted to breakdown stress drop $\tau_{s}-\tau_{d}$ by multiplication by the normal stress.

All the model parameters are considered to be functions of spatial coordinates along the fault. For computational feasibility we limit their potential variability along the fault by defining them as fields bilinearly interpolated from a relatively coarse grid of control points (see Figure 1c).

\subsection{Forward Problem}

To speed up the forward problem, we separate the calculation of synthetic waveforms into two steps: dynamic rupture simulation of the rupture propagation and evaluation of the representation integral using precomputed Green's functions (e.g., Ruiz \& Madariaga, 2011; Twardzik et al., 2014). In this way, we minimize the computational cost of the dynamic rupture simulation by carrying it on a computational domain limited to the close surrounding of the fault. Moreover, solving both dynamic rupture and wave propagation with the same finite difference method (with uniform grid size) would be a waste of resources, since the grid size required to resolve dynamic rupture is smaller than the one required to resolve wave propagation at the desired frequency. Details of the two steps are as follows.

In the first step, the dynamic rupture simulation is carried out on a dense grid of finite difference nodes (Figure 1c). The grid step size is controlled by the cohesive zone width in the dynamic simulations (Day et al., 2005). The input dynamic rupture parameters are bilinearly interpolated from a coarse grid of model control points onto the dense finite difference grid. The control point grid density is set according to preliminary experiments as a compromise between the number of parameters in the inversion and the rupture complexity required to fit observed data in the frequency range considered. In the second step, the resulting slip rates are spatially averaged onto the coarser grid of subfaults (Figure 1c) in which Green's functions have been precalculated and stored. This grid step size must be smaller than the minimum wavelength in the wave propagation calculation (Gallovič et al., 2015; Spudich \& Archuleta, 1987). Waveforms are then calculated following the representation theorem (Aki \& Richards, 2002), that is, by summing up contributions from the individual subfaults obtained as convolution of the individual averaged slip rates with the respective Green's functions. For the actual grid parameters considered in the present study see Table 1.

Despite the effectiveness of the described approach, we still need an extremely efficient dynamic rupture simulator. We employ finite difference code FD3D developed at UCSB and ENS by K. B. Olsen, R. Madariaga, and R. Archuleta (Madariaga et al., 1998; the code is available at http://www.geologie.ens.fr/ 
$\sim$ madariag/Programs/programs.html). The 3-D elastodynamic equation is solved in a uniformly discretized box covering the fault using a fourth-order staggered-grid velocity-stress method (Madariaga et al., 1998) with boundary condition applied on the fault to obey the slip-weakening friction law formulated in the thin fault approximation. The latter was introduced by Madariaga (2005) as an improved version of the thick zone approach of Madariaga et al. (1998), in which the inelastic zone is only one grid-step thick. According to Madariaga (2005), the thin-fault method is more accurate than the original thick-fault method tested by Dalguer and Day (2006). To speed up the calculations, the fault is considered to be vertical, aligned with one side of the computational box, where symmetry conditions are applied in order to solve the problem only on half of the volume. On the top of the box we prescribe free-surface boundary conditions by the stress-imaging technique (Graves, 1996; Kristek et al., 2002; Levander, 1988). On all the other sides we apply absorbing boundary conditions of the Clayton-Engquist type (Clayton \& Engquist, 1977). A one-dimensional layered elastic velocity structure is assumed. The actual fault we aim to simulate is not vertical as in the FD3D code. To partially correct for this difference, we vertically stretch the velocity model in order to respect the original along-dip position of the fault intersections with the velocity model layers. This way, we keep the elastic parameters and density along the vertical fault the same as if the fault had the true dip. Moreover, thanks to the relative simplicity of the FD3D code, it was straightforwardly ported to Graphics Processing Units (GPUs) using OpenACC directives, which are implemented in the Portland Fortran compiler. The additional speed up achieved by the GPU (NVIDIA GTX Titan) compared to a single-core CPU run is a factor of about 5. For obvious reasons the solution of the rupture simulation obtained by the FD3D code is an approximation to the true solution. While we document that our simplifications are adequate for our specific problem, it is beyond the scope of this paper to systematically evaluate their range of validity for more general cases. Nevertheless, readers can follow similar validation approaches as exemplified in our paper to judge if the assumptions are adequate for their own applications. This compromise is made to achieve a major speed up of the dynamic rupture simulation (see below). We emphasize that the typical high computational demand of more complete dynamic rupture simulation codes (e.g., Uphoff et al., 2017) prohibits their use for dynamic source inversion as formulated here.

Nevertheless, it is necessary to verify that the inverted model (or here the target model) is simulated sufficiently well by our simplified approach. We compare the rupture simulation calculated by FD3D and by the more advanced code WaveQLab3D (Duru \& Dunham, 2016), which has been verified in many community benchmarks of the SCEC/USGS Dynamic Earthquake Rupture Code Verification Exercise (Harris et al., 2018). WaveQLab3D simulates the first-order form of the 3-D elastic wave equation in collocated curvilinear grids. The mesh is discretized in space using high-order accurate finite difference schemes satisfying the Summation-By-Parts rule (sixth-order accurate central finite difference stencils in the interior with onesided third-order accurate boundary closures). The time discretization makes use of a fourth-order accurate low-storage Runge-Kutta approach. Boundary conditions and frictional interfaces are imposed weakly using penalties, resulting in a provably energy-stable scheme. WaveQLab3D handles fault boundary conditions as tractions at split nodes and uses perfectly matched layers as absorbing boundaries.

For this test we consider the Inv1 benchmark of the SIV initiative (Mai et al., 2016, Figure 2; see section 4 for more details). Figure 3 shows the along fault distribution of the dynamic parameters of the target model, which are defined on the dense 100-m FD grid. The WaveQLab3D simulation respects the actual fault geometry, dynamic normal stress changes, etc. but is $\sim 300 \mathrm{x}$ slower than the FD3D simulation: While WaveQLab3D requires $6 \mathrm{hr}$ on a recent eight-core CPU, FD3D requires only $10 \mathrm{~min}$ on a single-core CPU and $2 \mathrm{~min}$ on a GPU. Both WaveQLab3D and FD3D simulations use spatial resolution of $100 \mathrm{~m}$ (across the fault) and similar domain size.

Snapshots of the resulting rupture propagation are compared in Figure 4a. Figures $4 \mathrm{~b}$ and $4 \mathrm{c}$ show comparisons of the simulated final slip distributions and slip rates. The main features of the rupture propagation are well captured by the FD3D simulator. In the WaveQLab3D results the rupture is slightly faster owing to its better resolution of dynamic stress changes along the rupture front. In the WaveQLab3D results the peak slip rates are generally higher and the scalar seismic moment is larger by $3 \%$. Nevertheless, this difference does not affect significantly ground motions in our frequency range of interest due to the low-pass filtering (Figure 5). We note that the waveforms recalculated using WaveQLab3D fit perfectly the data downloaded from the SIV web server in the given frequency range. Importantly, all discrepancies between the FD3D and 
A)

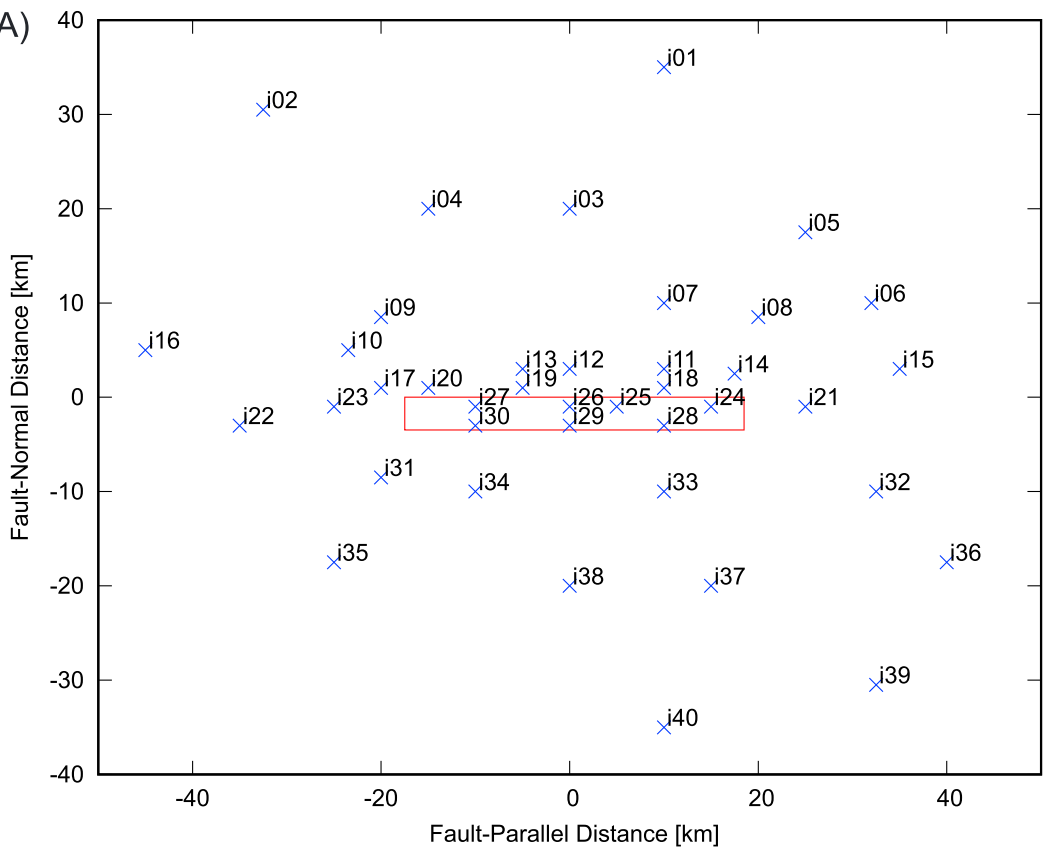

B)

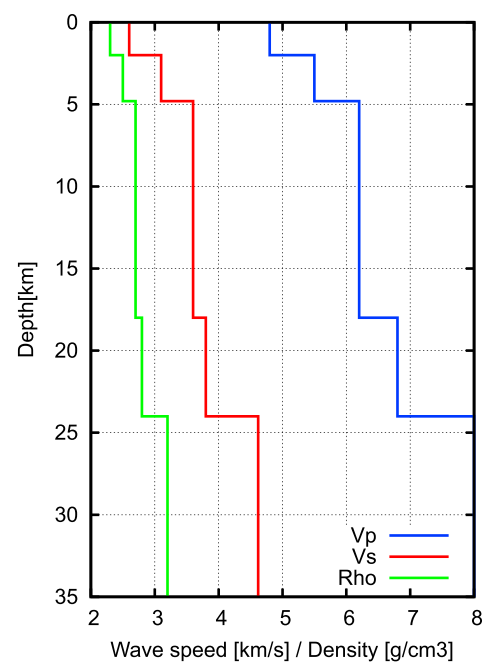

Figure 2. (a) Geometry of fault (red box) and station locations (crosses) of the source inversion validation (SIV) test Inv1. The right-lateral strike-slip fault dips at $80^{\circ}$ and reaches the surface. (b) Parameters of the 1-D elastic layered medium that is considered in calculation of the Green's functions.

WaveQLab3D results are smaller than the inversion uncertainties (quantified later); thus, for our purposes we judge that they are largely offset by the extreme gain in computational efficiency provided by the use of FD3D.

Synthetic Green's functions for a given set of stations (Figure 2a) are calculated by the Axitra code, which combines discrete wave number and propagator matrix methods (Bouchon, 1981; Coutant, 1989; Kennett \& Kerry, 1979). The 1-D velocity model is the one prescribed in the definition of the Inv1 benchmark (see Figure 2b). In Figure 5 we show a comparison of displacement seismograms computed from the FD3D
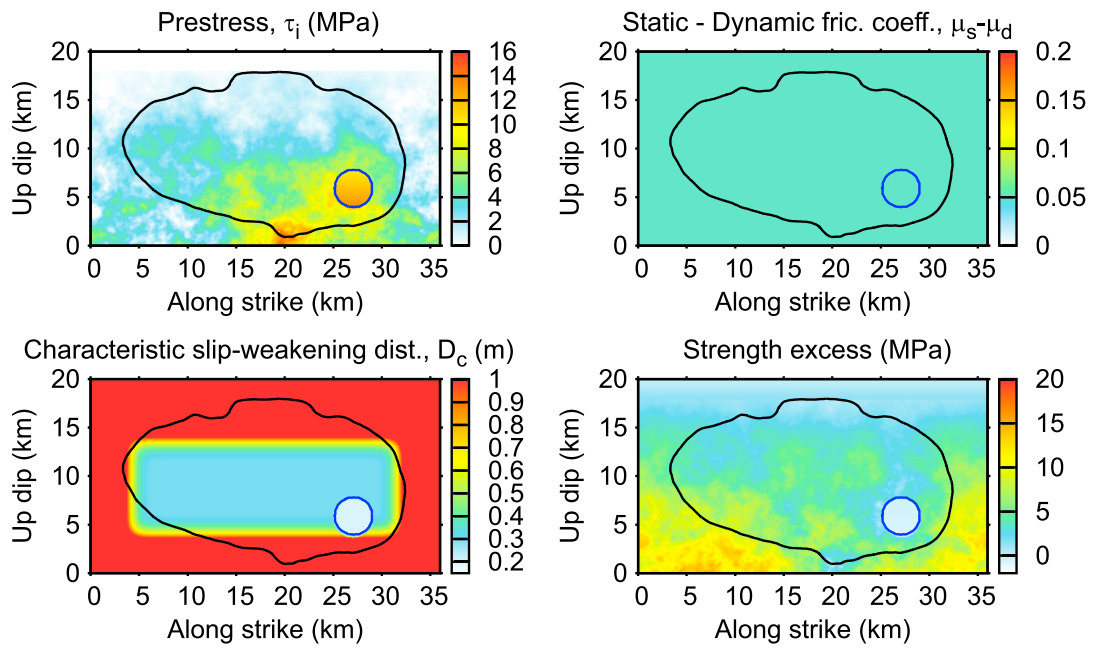

Figure 3. Input dynamic parameters of the target model of the Inv1 test, describing spontaneous dynamic rupture governed by a linear slip-weakening friction. The prestress is heterogeneous on the fault. Dynamic and static friction coefficients are considered homogeneous at 0.60 and 0.55 , respectively, that is, the friction drop being 0.05 . Nucleation region with negative strength excess is delimited by the blue circle. $D_{c}$ is constant at $0.3 \mathrm{~m}$ in approximately the center of the fault, while it is set to $0.2 \mathrm{~m}$ in the nucleation region and linearly increases to $D_{c}=5 \mathrm{~m}$ toward the fault boundaries. The black contour delineates the ruptured area (see Figure 4). 
A) WaveQLab3D
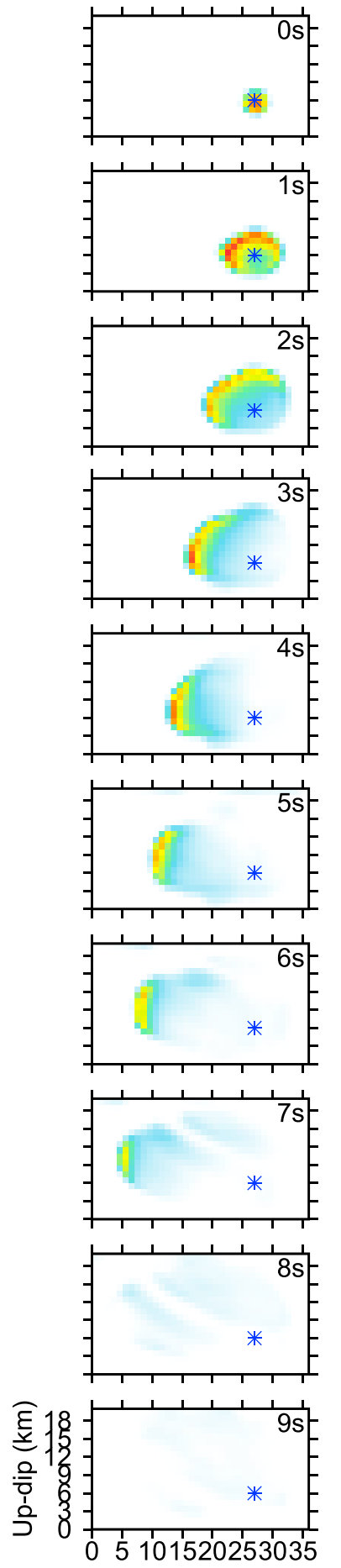

Along strike $(\mathrm{km})$
FD3D
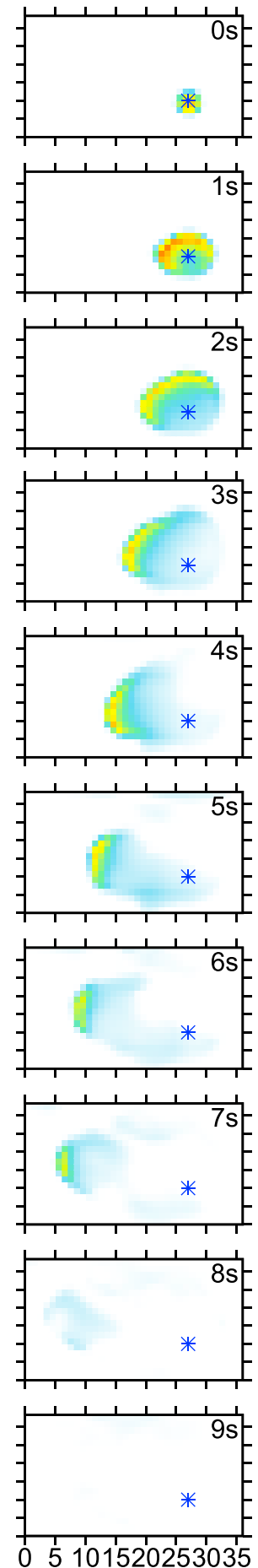

Along strike (km)
B)

\section{WaveQLab3D}

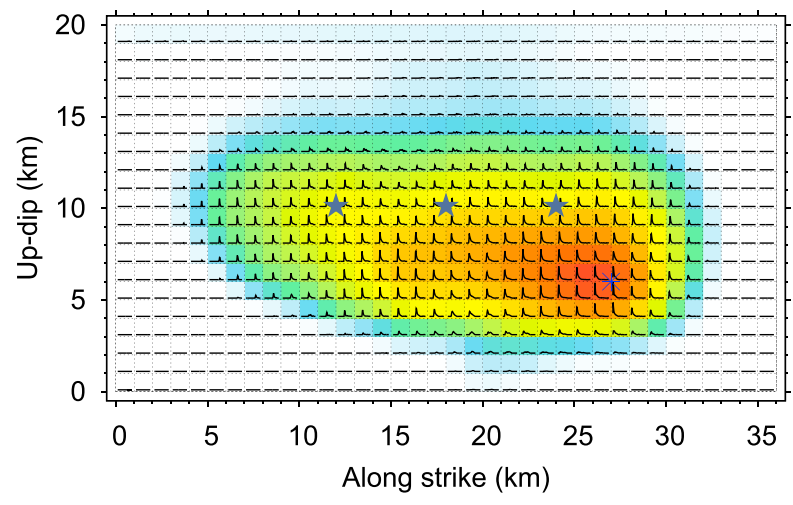

FD3D

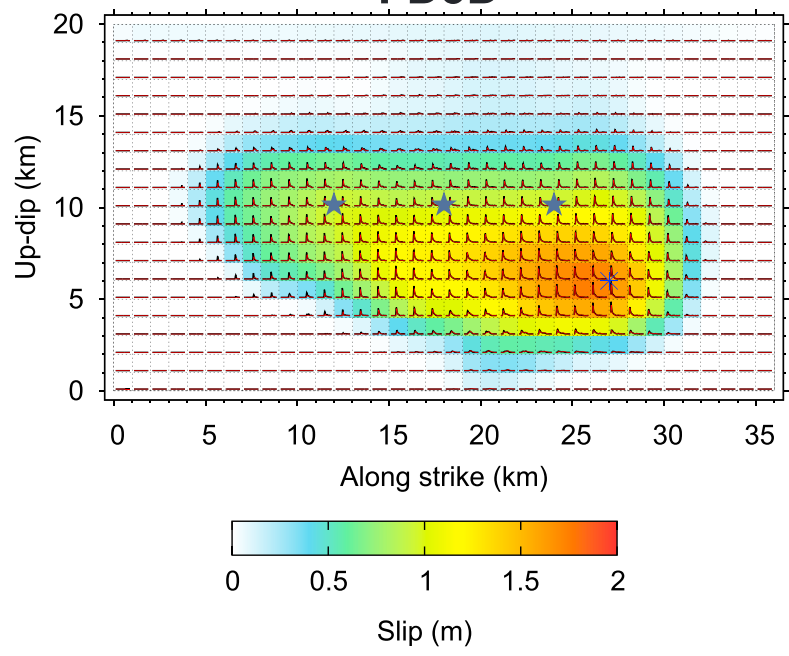

C)
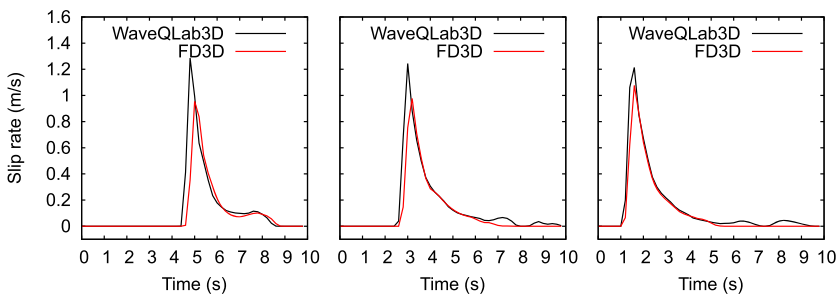

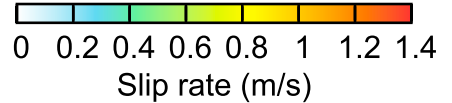

Figure 4. Spontaneous rupture propagation calculated by two different codes (see legend). In WaveQLab3D the dipping fault geometry is fully respected in the simulation, while in FD3D the fault is approximated by a vertical plane to speed-up the simulation. The simulation results are compared in terms of (a) slip rate snapshots, (b) slip and slip rates plotted along the fault, and (c) slip rates from three selected points depicted in panel (b). The results match almost perfectly, having model variance reduction in terms of the slip rate functions equal to 0.83 . Comparison of the corresponding waveforms is shown in Figure 5 . 


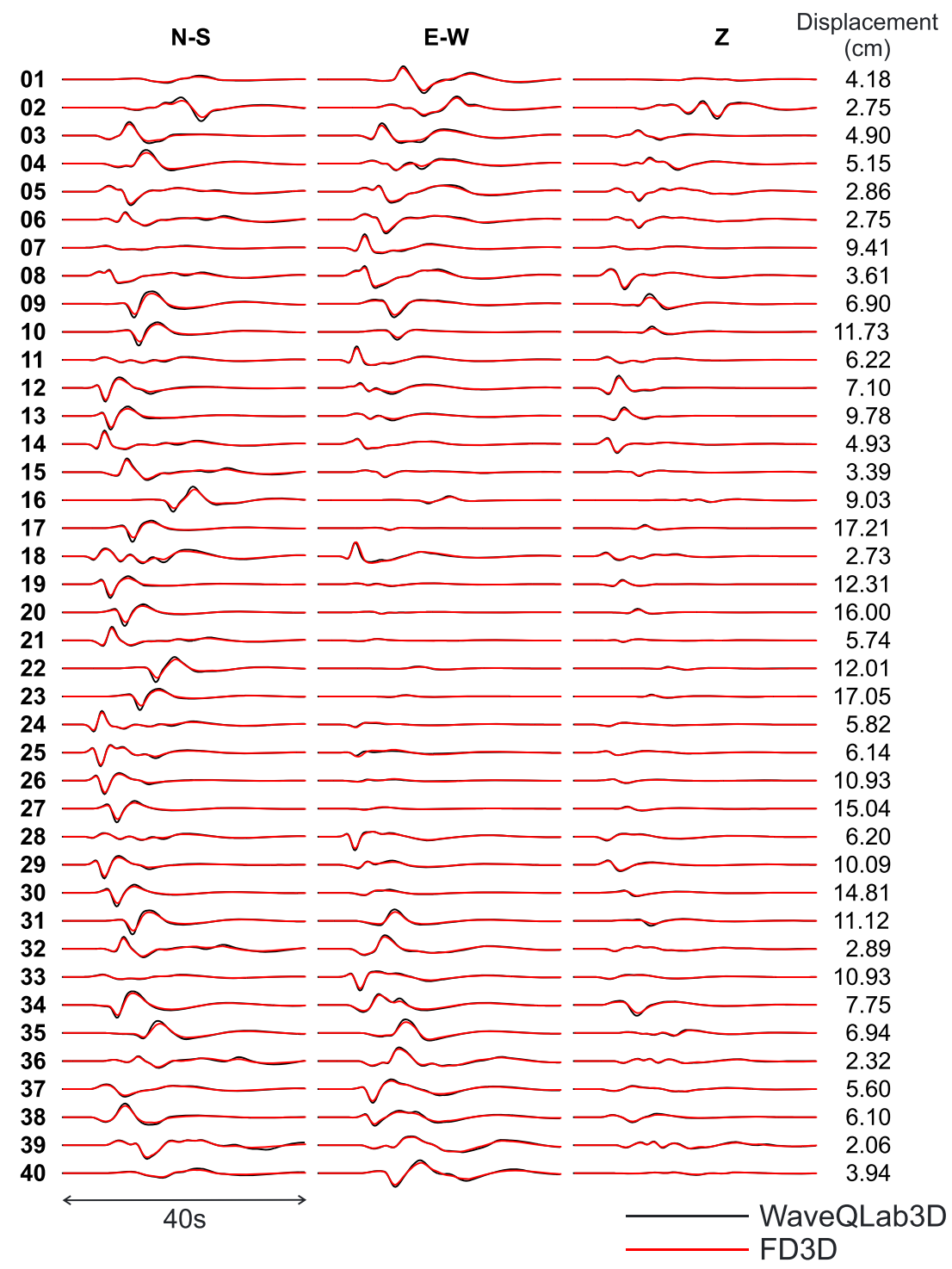

Figure 5. Displacement seismograms of the target model in frequency range $0.05-0.50 \mathrm{~Hz}$, as considered in the inversion, calculated by the two simulation methods (see legend). The station names are depicted on the left, while maximum amplitudes at the individual stations are shown on the right. The waveforms agree very well with variance reduction in the data space reaching 0.94 and with standard deviation of residuals (modeling error) being $0.5 \mathrm{~cm}$.

and WaveQLab3D results, assuming the dynamic parameters of the target model, in the frequency range $0.05-0.50 \mathrm{~Hz}$ that will be considered later in the inversion. The waveforms agree very well, the variance reduction (in the data space) reaches 0.94 .

\subsection{Sampling of the Posterior PDF}

MCMC methods are widely used in the Bayesian framework to sample the posterior PDF in model space (see, e.g., the review by Sambridge \& Mosegaard, 2002). The main advantage is that the set of acquired models represents samples randomly drawn following the posterior PDF. This set can be then directly used in a subsequent statistical analysis of the model uncertainty. We note that the statistical analysis does not have to be limited to the inferred dynamic rupture parameters but can be also applied to other derived quantities, such as the resulting kinematic rupture parameters.

Generally, the MCMC approach works as follows. In each step of the chain, the model parameters (prestress, friction drop and $D_{c}$ ) are randomly perturbed considering log normal PDFs. The new proposed model is 
checked against the priors. If its parameters exceed their prior bounds or if negative strength excess is present outside the prescribed nucleation area, the model is immediately discarded, without running the corresponding dynamic simulation, and a new model is generated. Otherwise, the dynamic simulation is run and the waveform misfit is evaluated (and thus the corresponding posterior PDF value of equation (2)). The proposed model is accepted or rejected based on the Metropolis algorithm: If the posterior PDF value of the proposed model is higher than that of the unperturbed one, the model is accepted; if the PDF value is lower, the model may be still accepted with probability given by the Metropolis-Hastings rule (Metropolis et al., 1953).

Here we use a quite novel modification of the MCMC method-the parallel tempering (Falcioni \& Deem, 1999; Sambridge, 2013; etc.). It is similar to simulated annealing, as the posterior PDF (equations (1) and (2)) is modified by a parameter temperature $T$ :

$$
p(\boldsymbol{m} \mid \boldsymbol{d}, T)=c_{1} p(\boldsymbol{m}) \exp \left(-\frac{1}{T} \frac{1}{2} \sum_{i=1}^{N} \frac{\left\|\boldsymbol{s}_{i}(\boldsymbol{m})-\boldsymbol{d}_{i}\right\|^{2}}{\sigma_{i}^{2}}\right) .
$$

The higher is the temperature, the smoother is the modified PDF, so that the Markov chains at higher $T$ can more easily skip local minima thanks to a larger likelihood of acceptance of the advance step. Unlike in simulated annealing, where temperature $T$ gradually decreases in an ad hoc way, in the parallel tempering method a set of Markov chains with different temperatures advance through the model space. To ensure sufficient sampling of the model space, the chains are allowed to exchange temperature values following a modified Metropolis-Hastings rule. Samples of the posterior PDF are then obtained from chains at temperature $T$ $=1$. In a synthetic example, Sambridge (2013) showed that the tempered chains may converge at least 10 times faster than the nontempered MCMC approach.

\section{Application to a Synthetic Test}

The SIV initiative formulated a set of community benchmarks to assess the performance of earthquakesource inversion methods and to understand strengths and weaknesses in determining models and their uncertainties (Mai et al., 2016, http://equake-rc.info/SIV/). Although initially the tests were defined for kinematic inversions, we take advantage of the fact that the first benchmark, Inv1, is based on simulation of a crack-like spontaneous dynamic rupture, embedded in a layered isotropic velocity-density structure. Waveforms to be inverted were calculated on a set of stations (Figure 2a) assuming a 1-D velocity profile (Figure 2b). Note that the aim of our dynamic inversion is not only to infer dynamic parameters along the fault but also to test the performance of the frictional model as a constraint to regularize the inverse problem.

\subsection{Target Model and Waveforms}

The Inv1 benchmark target model is a strike-slip rupture on a fault plane dipping at $80^{\circ}$ and reaching the surface, with dynamic parameters distributed as displayed in Figure 3. While the prestress is variable along the fault, the dynamic and static friction coefficients are constant at 0.60 and 0.55 , respectively; the friction drop is 0.05 . The characteristic slip-weakening distance $D_{c}$ is essentially constant, $0.3 \mathrm{~m}$, in the region where the rupture takes place (approximately in the center of the fault), and it linearly increases to $5 \mathrm{~m}$ toward the fault boundaries to ensure smooth rupture termination. The rupture starts from a small nucleation region with negative strength excess and $D_{c}=0.2 \mathrm{~m}$ (see Figure 4). The final seismic moment $M_{0}$ is approximately $1 \times 10^{19} \mathrm{Nm}\left(M_{w} 6.6\right)$. As data for the inversion, we use displacement waveforms filtered in the frequency range $0.05-0.50 \mathrm{~Hz}$ (see Figure 5).

\subsection{Inversion Setup}

In the inversion we use exactly the same fault plane geometry, spatial and temporal discretization, and Green's functions as when generating the benchmark data. We pretend we only have an approximate knowledge of the position of the nucleation, which serves as one of the prior constraints. For the complete list of assumed priors see Table 1 . The number of model control points where the three model parameters $\left(\tau_{i}, \mu_{s}-\mu_{d}, D_{c}\right)$ are defined is $13 \times 9$, which makes a total of 351 unknowns. The effective number of 
model parameters is about $1 / 4$ lower because those that are located outside of the ruptured area are unconstrained.

For the Bayesian inversion we need to specify modeling and data errors (equation (2)). Here the modeling error can be represented by the differences between target model synthetics as calculated by WaveQLab3D and FD3D (Figure 5). The standard deviation of the residual seismograms is $0.5 \mathrm{~cm}$. We here assume that the data error $\sigma_{i}$ in equation (2) is much larger, $10 \mathrm{~cm}$. Such value is in the range what is considered in real data applications, where $\sigma_{i}$ is comparable with the amplitudes of the observed data mainly due to the imperfect knowledge of crustal structure (Hallo \& Gallovič, 2016; Sokos \& Zahradník, 2013; Yagi \& Fukahata, 2011) and imprecise location and geometry of the fault (Ragon et al., 2018, 2019).

In an ideal situation the MC Markov chains are started from randomly selected models and allowed to explore the model space automatically for a very long time. Due to the limited computational resources and the complexity of the posterior PDF, for example, having vast no-rupture areas, we modified the automatic procedure to accelerate the convergence to reasonable solutions as briefly described in the following.

Before proceeding to the actual dynamic inversion, we built a plausible model with a decent data fit to serve as an initial guess. We started from a slip model obtained by linear kinematic inversion using the code LinSlipInv (Gallovič et al., 2015; Pizzi et al., 2017; Sokos et al., 2016). The stress drop distribution, $\Delta \tau$, was computed from the slip model using the static dislocation solutions in a homogenous half space by Okada (1992). The resulting stress drop was considered as an initial model for prestress above the dynamic stress by setting $\tau_{i}=\Delta \tau$. Then the other parameters were manually adjusted to obtain rupture propagation from the hypocentral area without reaching the surface. The latter required to set $D_{c}$ to larger values in the upper $\sim 5 \mathrm{~km}$ (we note that this does not prevent the inversion performed further from finding models breaking the surface because the $D_{c}$ values at the shallow depths are still part of the inversion). At this point the data fit was only decent; its variance reduction (VR) was slightly above zero. Then we used the parallel tempering approach to allow the model to improve the waveform fit. Prior limits considered on the values of the dynamic parameters are specified in Table 1 . Since at this point we needed a huge amount of models to be tested, as the PDF is rather flat far from the optimum model, we used a twice coarser discretization in the FD3D code (leading to 16 times faster calculations).

Once VR increased to $\sim 0.8$, we switched to the discretization specified in Table 1 and let the parallel tempering explore the model space for a much longer time. We stopped the inversion several times, selected the actual best fitting model, and used it as a new starting model for the next iteration of the inversion. Once VR reached values larger than 0.9, we started the final and most extensive exploitation of the model space (Sambridge \& Mosegaard, 2002). Overall, we used 10,000 MCMC chains with randomly distributed temperatures up to 100 , where about $1 / 5$ were at temperature 1 . All the chains visited a total of $\sim 1,000,000$ models. Every tenth step of the Markov chain the models at $T=1$ were saved. The final set consists of $\sim 5,000$ model samples representing the random draws from the posterior PDF. This was achieved by combining five 14 days long runs on Xeon supercomputer cluster IT4I, where we utilized 200 CPUs, each running 10 temperature levels sequentially, and our local farm of $10 \mathrm{GPU}$ cards. This makes our approach very feasible with standard present-day resources.

\subsection{Inversion Results}

Figure 6a shows histograms of data VR for the final ensemble of $\sim 5,000$ samples representing the posterior PDF. The VRs range from 0.9 to 0.97 of the best fitting model. In the following we consider only models having a posterior PDF value larger than $0.1 \%$ of the PDF maximum, to ignore model samples that do not fit the data sufficiently. This way we end up with an ensemble of so-called accepted models whose distribution of VR is shown in the red histogram of Figure 6a.

Since the aim of the synthetic test is to find models that are close to the target model, we evaluate for each model sample its so-called model VR that expresses the differences between the model slip rate functions and those of the target model, after the underdiscretization to the Green's functions subfault grid (see Table 1 and Figure 1c). The resulting model VRs are shown as histograms in Figure 6b. The values range from -0.1 to 0.7 . We note that the model that best fits the target data has a data VR of 0.97 and a model VR of 0.3. Conversely, the rupture model with the largest model VR (0.71) has a data VR of 0.94. This 

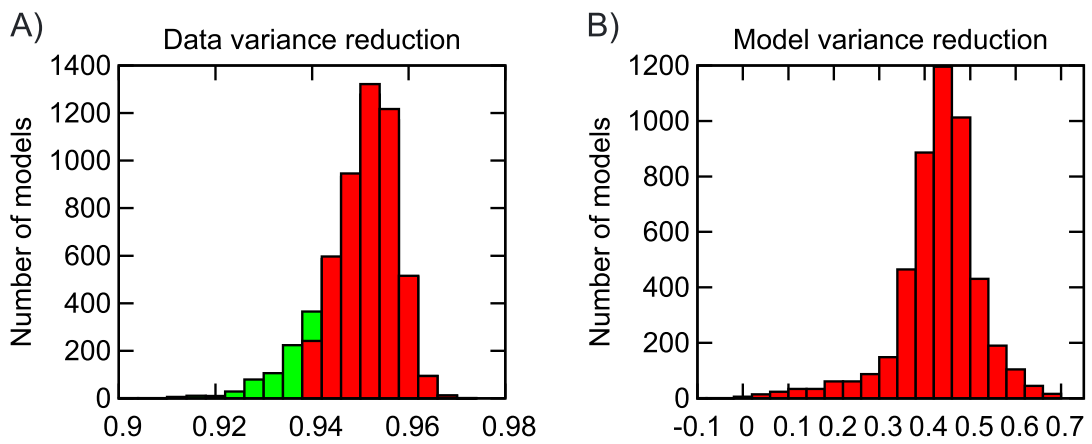

Figure 6. (a) Histograms of variance reduction (VR) in the data space of all posterior model samples (red + green); red histogram accounts only for models having posterior probability density value larger than $0.1 \%$ of the posterior PDF maximum (accepted models). (b) Histogram of model VR for the accepted models expressing similarity with the target model in terms of the slip rate functions. Note that the model that best fits the target data has model VR as low as 0.3 with the target model. Rupture model with the largest model VR (0.71) is explored in Figure 7.

suggests that the best fitting solution is biased, both due to the use of a diverse parameterization of the target and inverse models and the use of less precise forward solver. We note that in real data applications the bias will be even stronger mainly because of imperfect knowledge of crustal structure and fault geometry (e.g., Hallo et al., 2019; Hallo \& Gallovič, 2016; Ragon et al., 2018, 2019). Properties of the model with the largest model VR and the data VR are examined later on.

Figure 7a compares the slip rates of the target model with those of the model sample that is closest to the target model in terms of the slip rates, that is, with the highest model VR. The slip rates agree very well, especially in the high-slip region. For this model sample the rupture, however, stops somewhat earlier on the left side of the fault. Nevertheless, as demonstrated later in Figure 9b, there are other models in the posterior ensemble, for which the rupture propagates even further than the target model. We highlight that our approach does allow spontaneous, nonprescribed rupture stopping, which was identified as one of the biggest challenges in previous dynamic source inversion approaches (e.g., Madariaga \& Ruiz, 2016).

We now compare the inferred dynamic rupture parameters, the main outcome of the present inversion. For the model discussed above, Figure $7 \mathrm{~b}$ shows the along-fault distribution of prestress, friction drop, $D_{c}$, and strength drop (the product of the friction drop and the depth-dependent normal stress). These plots are to be compared with those of the target model shown in Figure 3. The dynamic parameters inferred outside the ruptured area (at the fault edges) are essentially unconstrained: obviously, such areas do not affect the rupture propagation, thus have no effect on the radiated wavefield, and are inaccessible to the inversion. Consequently, the uncertainty of the dynamic parameters in such areas is rather large as discussed later.

The prestress values are correctly largest in the hypocentral area. Also, within $10-5 \mathrm{~km}$ to the left along strike they are well determined. Nevertheless, farther away and also at shallower depths, the prestress is overestimated, while in the deepest part the prestress misses the locally elevated values of the target model. The friction drop of this particular model sample exhibits pronounced heterogeneity although in the target model this parameter is constant along the fault. The inferred values range between 0.03 and 0.1 , that is, around the true value of 0.05 . The distribution of $D_{c}$ of the discussed model is also heterogeneous along the fault, although to a lesser extent than the friction drop. While in the target model $D_{c}$ is smaller only in the nucleation patch, in the inverted model sample $D_{c}$ is smaller over a larger area in the nucleation region. This is perhaps compensated by the patch of overestimated values of $D_{c}$ near the middle of the fault. The large value of $D_{c}$ in the uppermost part is perhaps a remnant from the initial inversions performed to find a good starting model for the final round of the MCMC inversion (see section 4.2). The large values of $D_{c}$ at the bottom of the target model are not captured in the model sample. The inverted strength drop exhibits a slightly larger nucleation patch with larger overstress than prescribed in the target model. Farther away from the nucleation the strength excess is relatively well resolved, perhaps with somewhat overestimated heterogeneities at 
A)

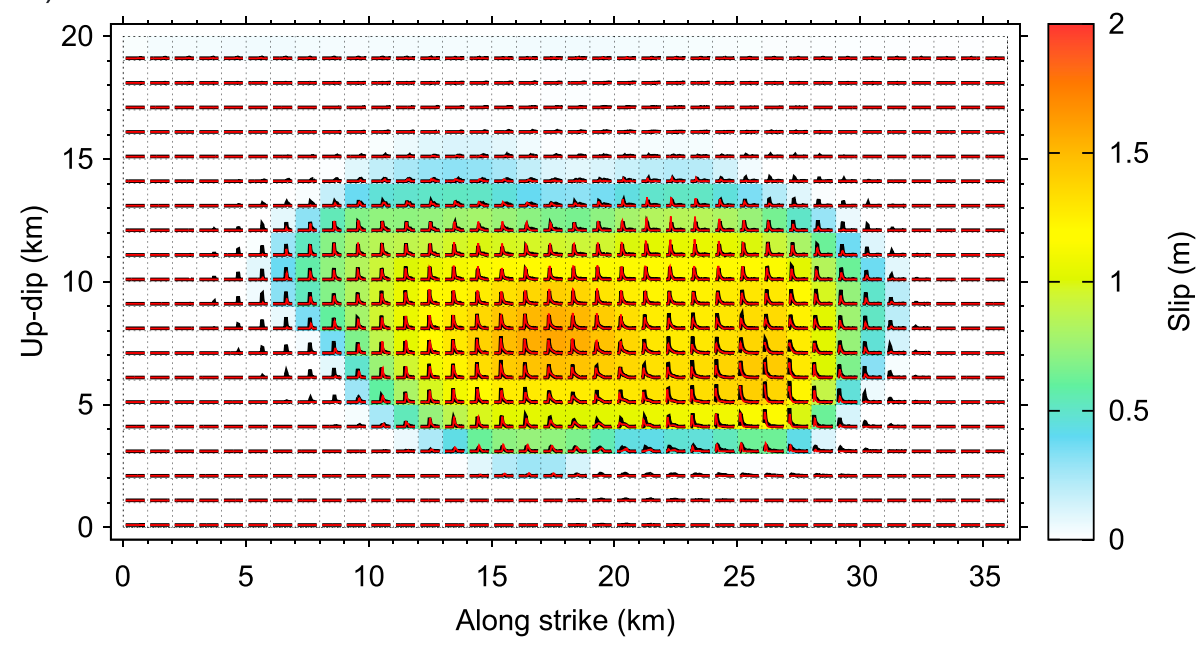

B)
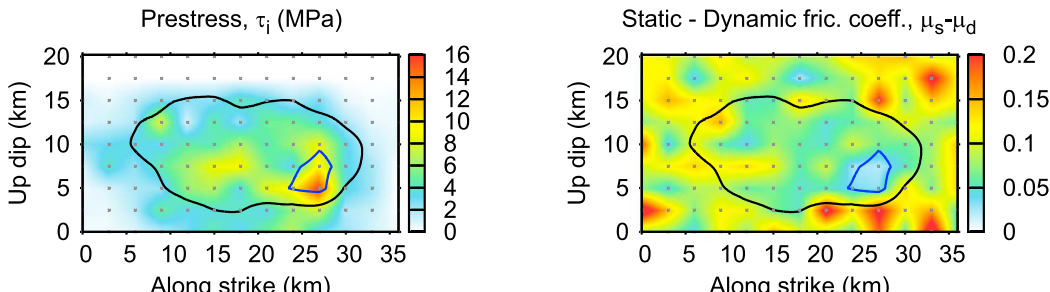

Characteristic slip-weakening dist., $D_{c}(m)$
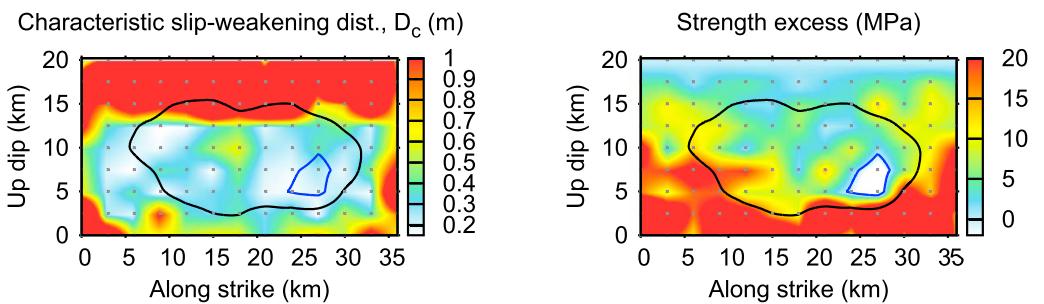

Figure 7. Properties of the inverted rupture model with the largest model VR. (a) Slip distribution (color coded) with superimposed slip rates (in red). The black slip rates in the background correspond to the target model for reference. (b) Dynamic rupture parameters of this particular inverted model. Black and blue contours delineate the slip distribution and nucleation zone (having negative strength excess), respectively. Gray dots denote positions of the model control points. Data VR of the present model is 0.94 .

the edges of the ruptured region. To summarize, the dynamic parameters of this particular model sample seem to be well resolved in the area of the largest slip, while some disagreement appears toward the edges of the slip distribution.

Figure 8 shows properties of the maximum a posteriori model, that is, the inverted rupture model with the largest data VR (0.97). It overall exhibits similar properties as the previously discussed model. However, positions and amplitudes of the small-scale heterogeneities of the individual parameters vary in most cases, see, for example, the missing large $D_{c}$ heterogeneity in the middle of the rupture, or the larger friction drop ibid. This variability among the models illustrates the inherent uncertainty of the solution, calling for inspection of the inverted model ensemble in a statistical sense.

Indeed, the above described properties of the inverse solution were related to single-sample models drawn from the full posterior PDF. However, in the Bayesian approach we should interpret the results of the inversion by analyzing the whole ensemble of model samples to take into account the inherent uncertainty. For this reason, Figure 9a displays the main rupture parameters (slip, stress drop, and rise time) along the fault averaged over the accepted solutions (middle column) and their uncertainty quantified as 2 times the 
A)

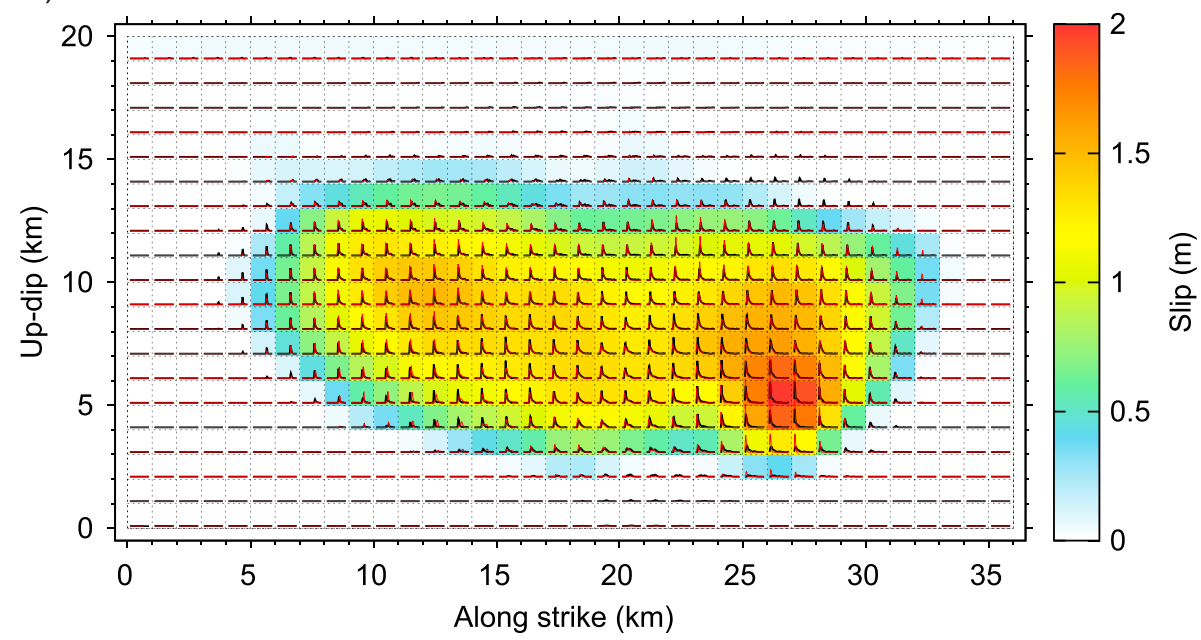

B)
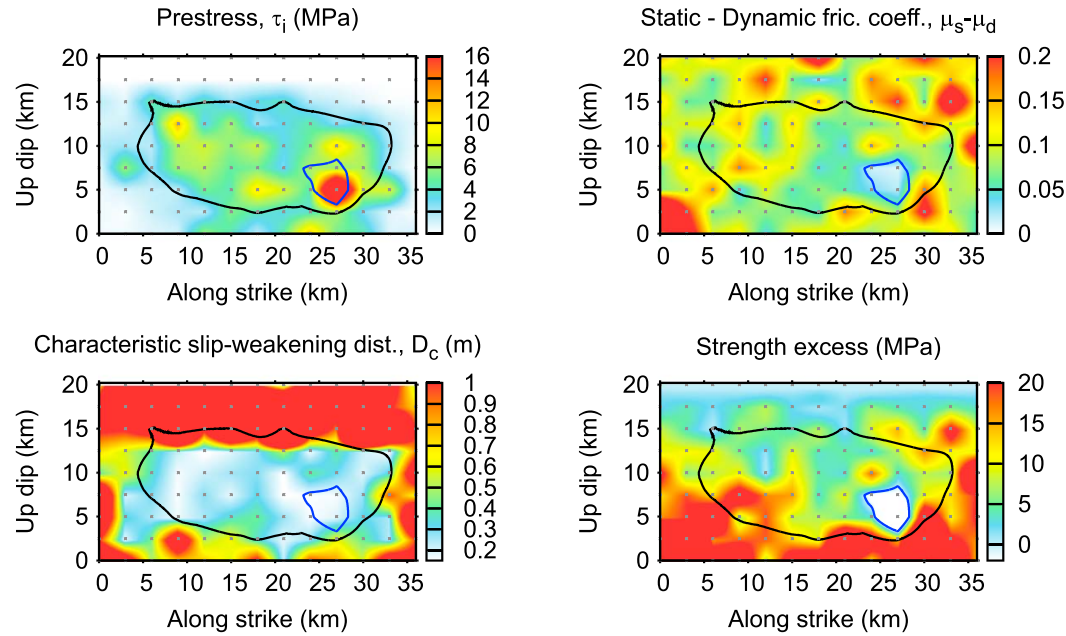

Figure 8. Same as Figure 7 but for the inverted rupture model with the largest data VR (0.97).

standard deviation (2 sigma, right column), compared with those of the target model (left column). The main characteristics of the target model are captured by the mean model, such as the basic shape of the main slip patch, largest stress drop in the hypocentral area, and rise times within the main slip patch. Nevertheless, some details of the target model are not present in the mean model. For example, in the mean model the larger slip patch extends more to the left, the slip of the target model seems to reach shallower depths, and the localized relatively large values of rise times in the target model are less visible in the mean model. However, when taking into account the uncertainty of the models (right column), most of these apparent discrepancies are actually well within the estimated errors. For example, the large values of rise time present in the target model but missing in the mean model correspond to areas with large slip uncertainty.

The uncertainty of the actual rupture extent is displayed in Figure 9b, showing slip contours of all the accepted models. The uncertainty is smaller than the variability among models inferred by various modelers as shown in Mai et al. (2016). For a few model samples the rupture continues slightly to the surface and also to the bottom of the fault as in the target rupture model. The elevated uncertainties along the contour of the mean slip and stress drop (Figure 9a) are related to the variability of the fault rupture extent in the ensemble of accepted models (Figure 9b). The overall uncertainties of the rupture parameters within the slip patch are approximately $\sim 10 \%$ of the average values. 
A)
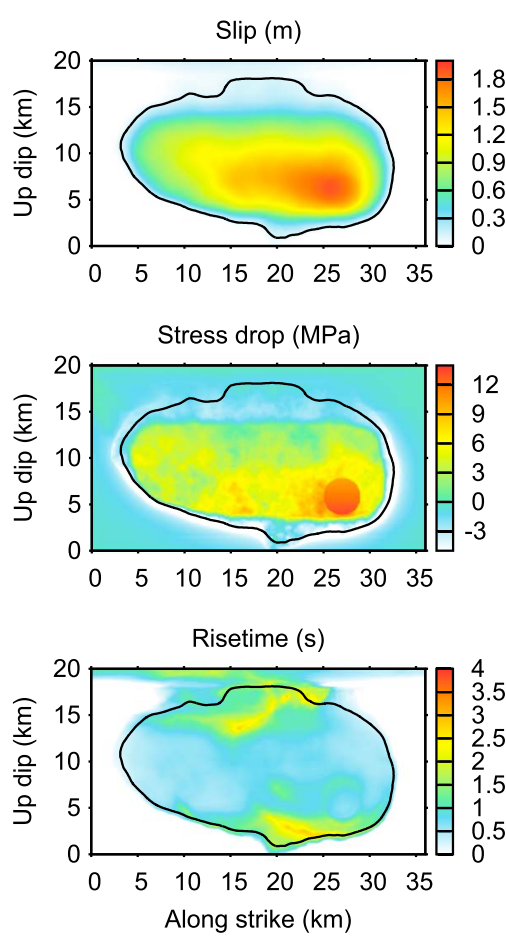

Inferred model mean
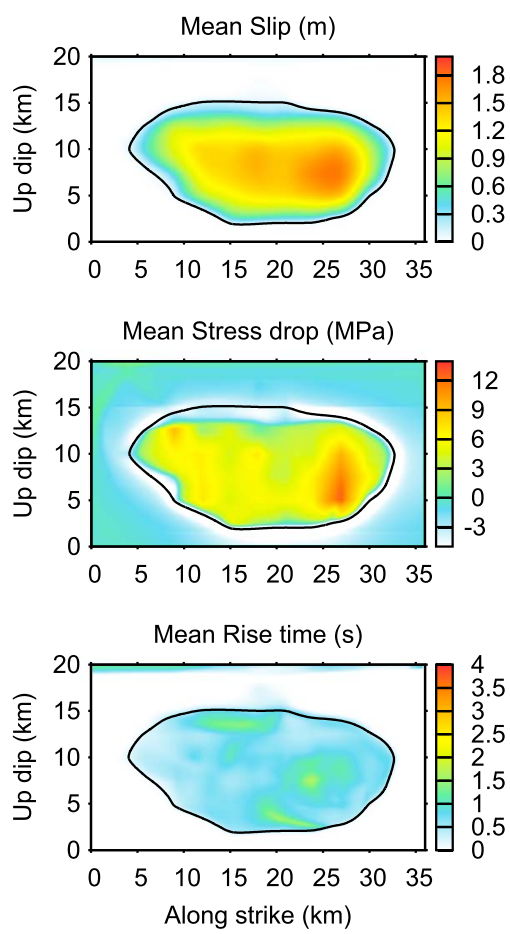

\section{Inferred model uncertainty}
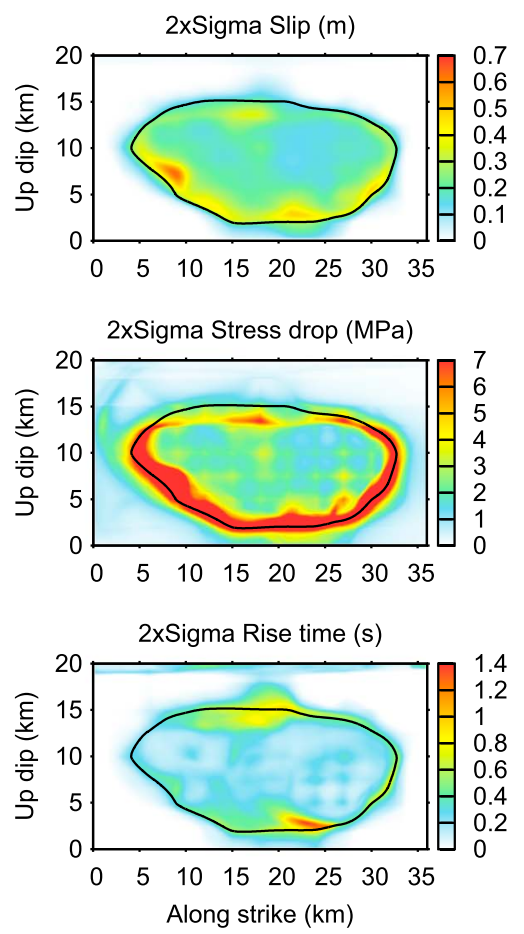

B)

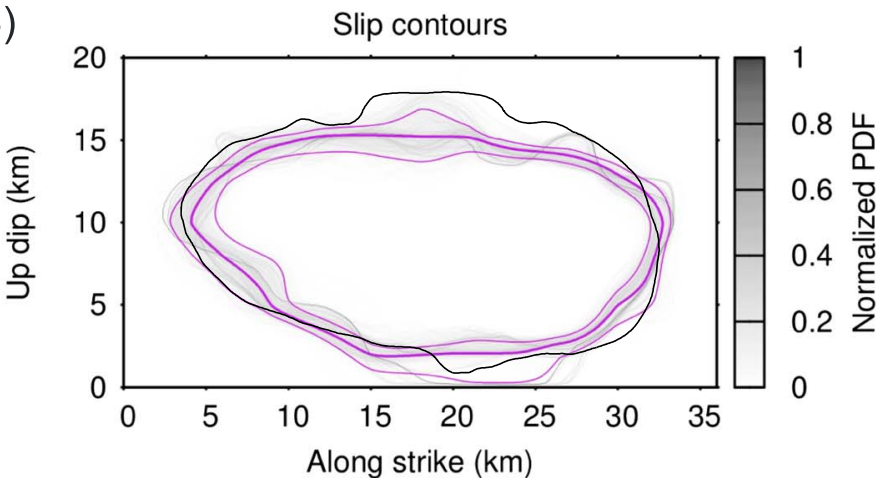

Figure 9. (a) Comparison of target model parameters along the fault (left column) with averaged model parameters over all accepted posterior samples (middle column) and the model parameters' uncertainty in terms of two sigma. (b) Slip contours of all accepted posterior model samples coded in gray displaying the variability of the inferred spatial rupture extent. The thick and thin magenta lines show the contours of the averaged slip model and its two sigma uncertainty, respectively. The black contour is the slip outline of the target model.

Figure 10 compares the dynamic parameters of the target model (left column) with the ensemble averaged values (middle column) and their uncertainties quantified as twice the standard deviations (right column). The distribution of the averaged prestress resembles that of the target model in the nucleation area and its vicinity within $\sim 10 \mathrm{~km}$. At shallower depths the averaged prestress is overestimated, while the higher prestress patch in the bottom of the target model is underestimated. The prestress uncertainty seems approximately constant along the slip patch, being largest in the nucleation area. The friction drop, which is constant in the target model, seems to increase outward from the nucleation area. Unlike the almost constant prestress uncertainty, the uncertainty of the friction drop increases from the nucleation area toward the edges of the slip patch, balancing the bias of the overestimated average values. The averaged distribution of strength excess captures the presence of increased values around the left, right, and bottom edges of the main slip patch, but the values are rather overestimated. Nevertheless, this is also balanced by the estimated uncertainty. The distribution of $D_{c}$ seems less heterogeneous than it is the case of the single PDF 


\section{Target model}
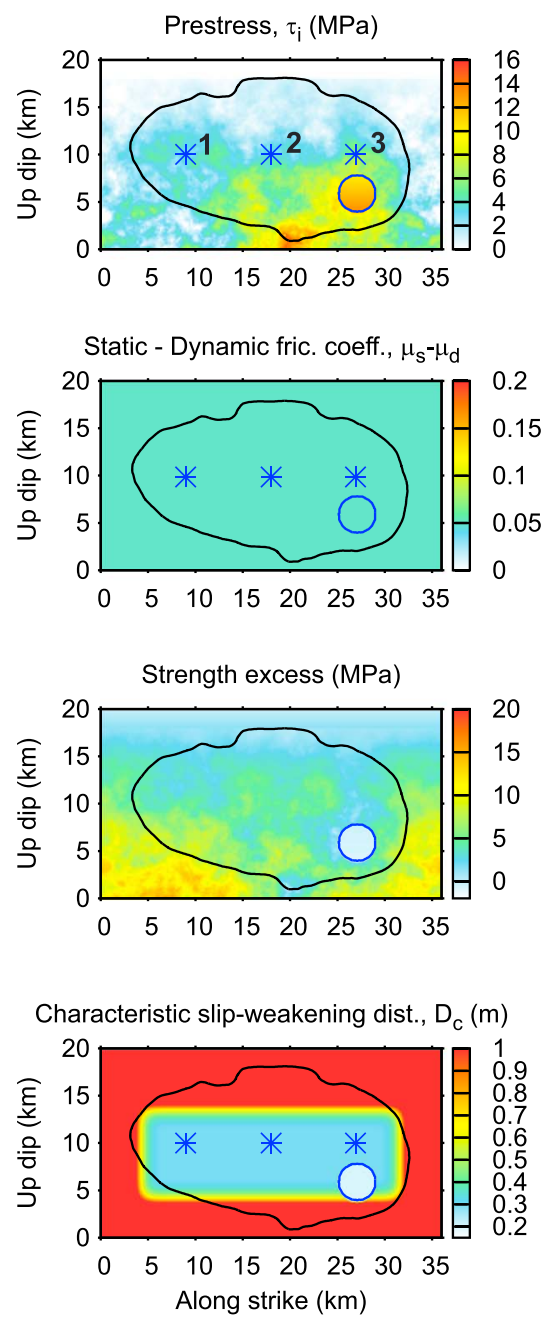

Inferred model mean
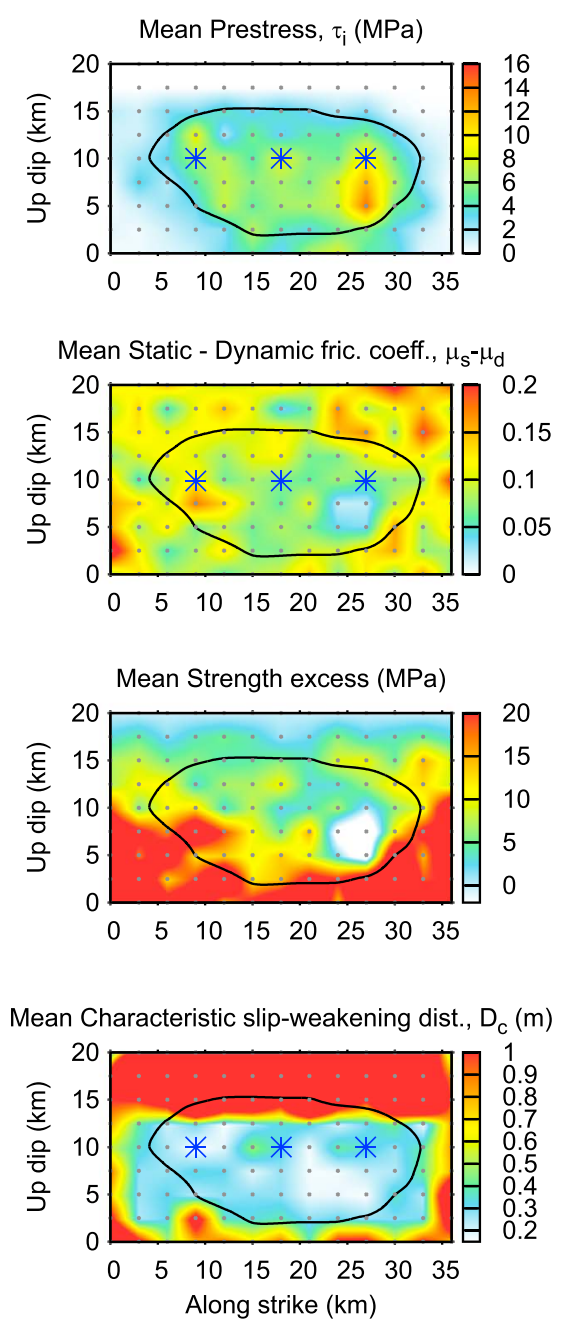

Inferred model uncertainty
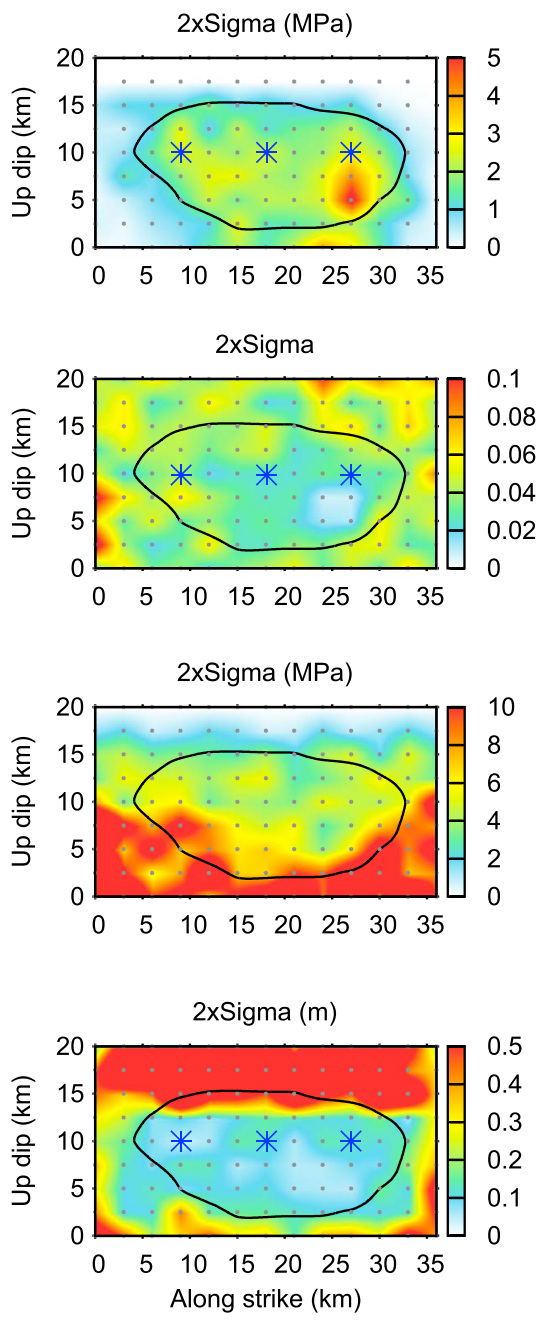

Figure 10. Comparison of target model dynamic parameters along the fault (left column) with distributions of dynamic parameters averaged over all accepted posterior samples (middle column) and their uncertainty in terms of two sigma. Black contour delineates the averaged distribution of slip (see Figure 9). Gray dots denote positions of the model control points.

samples of Figures 7 and 8. The remaining locally elevated values of $D_{c}$ in the averaged model are collocated with the areas of larger uncertainties. At the bottom edge of the slip patch the large $D_{c}$ values present in the target model are not very well recovered by the inferred models even when taking the uncertainty into account.

Additional insight can be gained from analysis of posterior marginal distributions at given positions on the fault, representing the local uncertainty of the inferred model parameters. Figure 11 shows such histograms for three points lying in the ruptured area (see Figure 10 for the position of the points). In all cases the true values of the target model have been visited, although they are in some cases at the tails of the histograms. This bias is further discussed in section 4.1.

We can conclude that the dynamic parameters are generally well resolved in the areas of large slip. The source parameters averaged over the ensemble are relatively smooth, having relatively large uncertainty (Figures 9-11), which is due to the local variability of the individual solutions (Figures 7 and 8). The bias of the dynamic parameters increases toward the edges of the slip distribution, which is, however, compensated by the similarly increasing uncertainties. This suggests that the target model values are inferred well within the estimated error margins, perhaps with the exception of the prestress and $D_{c}$ values at the bottom edge of the rupture. The latter can be ascribed to the fact that our posterior PDF sampling is still not perfect 


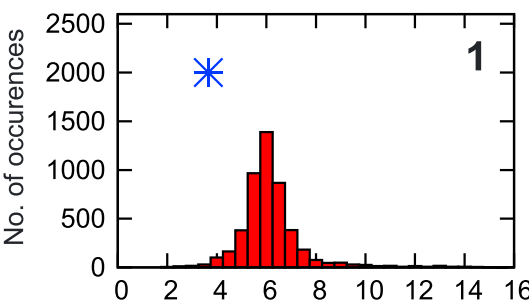

Prestress, $\tau_{\mathrm{i}}(\mathrm{MPa})$
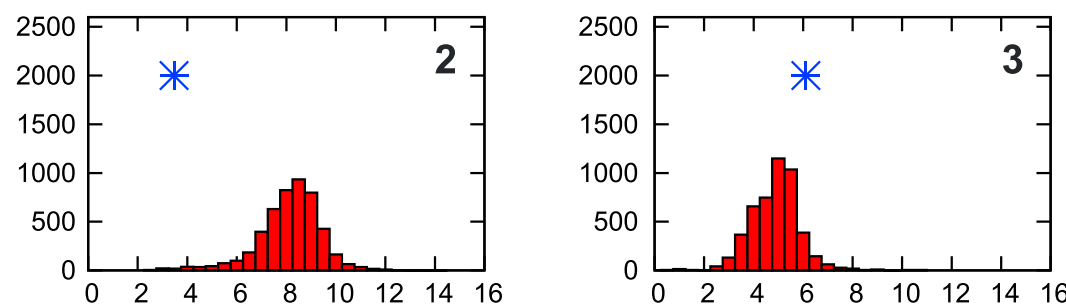

Static - Dynamic fric. coeff., $\mu_{\mathrm{s}}-\mu_{\mathrm{d}}$
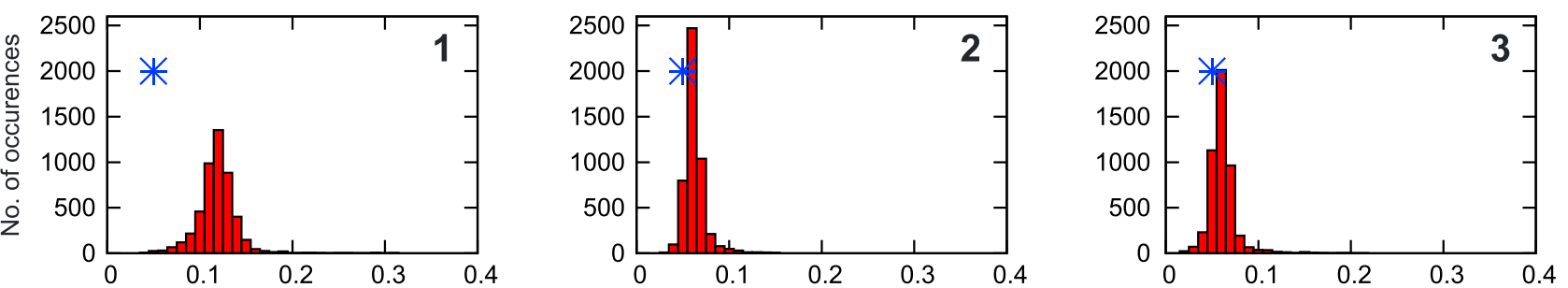

Characteristic slip-weakening dist., $D_{c}(m)$
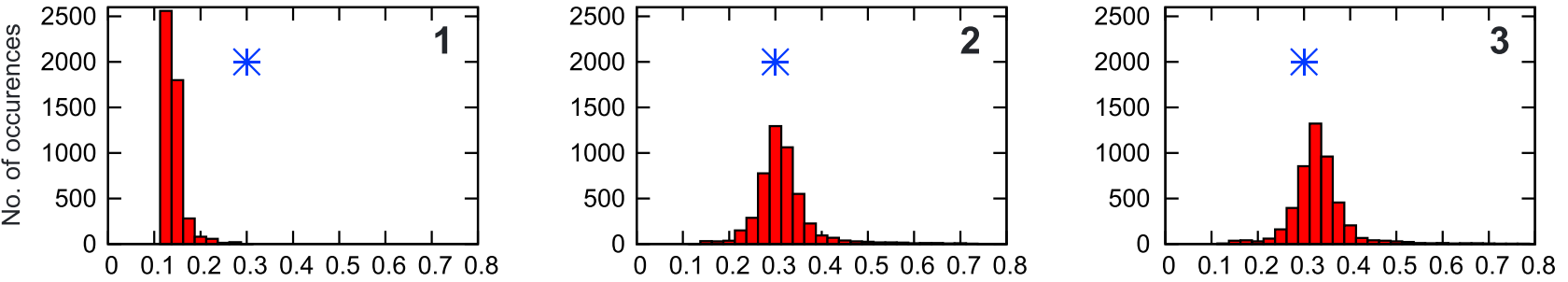

Figure 11. Histograms of model parameters at three selected points (see Figure 10 for their position) representing the posterior marginals. Blue stars represent true values from the target model.

due to the high dimensionality of the explored model space. Overcoming this challenge would require abundant computational resources and even more efficient forward solver. We note that the overall

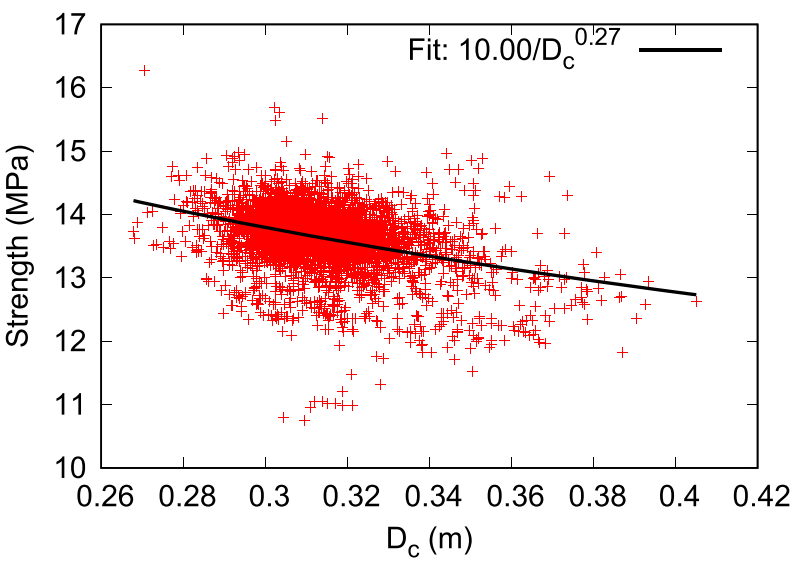

Figure 12. Slip-weighted mean strength $\tau_{s}$ versus slip-weighted mean characteristic slip-weakening distance $D_{c}$ for all accepted model samples (red crosses). Regression line fitting power law function is shown in black (see legend for the parameters). The obtained relation does not follow the expectation of the theoretical solution for the crack tip equation of motion in 2-D (Freund, 1990), indicating that the dynamic rupture growth is controlled by fracture energy, thus suggesting $\tau_{s} \sim 1 / D_{c}$. relative uncertainty of the dynamic parameters within the slip patch reaches $\sim 50 \%$ of the averaged values and is thus larger than the uncertainty of the kinematic rupture parameters.

\section{Discussion and Conclusions}

In this paper we have introduced a new computationally feasible dynamic source inversion approach, assuming a linear slip-weakening friction law. The method consists of two major components: (i) a direct solver FD3D (Madariaga et al., 1998), which, for given distribution of the dynamic source parameters along the fault, solves the rupture propagation and predicts the data that would have been observed by the receivers and (ii) a MCMC Parallel Tempering module (Sambridge, 2013) that samples the posterior Bayesian PDF in the model space, providing an ensemble of suitable models. Assuming a linear slip-weakening friction law, the inverted, spatially variable dynamic parameters are prestress, friction drop (difference between static and dynamic friction coefficients), and characteristic slip-weakening distance $D_{c}$. The rupture nucleation represented by areas with prestress higher than the strength (i.e., negative strength excess) is an inherent part of the inversion as the prestress and strength are treated independently. The nucleation area is just constrained to be contained in 
a prescribed area. For more efficient model space exploration we start the MCMC sampler from a dynamic model estimated from a preliminary kinematic inversion result.

The viability of the approach has been demonstrated on the example of the Inv1 benchmark of the SIV initiative (Mai et al., 2016). This benchmark is based on a spontaneous dynamic rupture with inhomogeneous distribution of prestress and $D_{c}$ and constant friction drop. Thanks to the efficient implementation of the inversion we were able to visit more than a million of models during the Monte Carlo Markov Chain procedure. The final set consists of $\sim 5,000$ model samples representing the random draws from the posterior PDF. Perhaps with the exception of underestimated values of the prestress and $D_{c}$ in the bottommost part of the fault, we can conclude that the dynamic parameters are generally well resolved in the area of large slip.

Our Bayesian approach results in a set of models that fit the observed data equally well. These models can be inspected for uncertainty of both the kinematic and dynamic parameters. In general, the dynamic parameters have larger uncertainty than the kinematic ones. This is not surprising, keeping in mind that the dynamic parameters are related, in a broader sense, to spatial derivatives of the kinematic parameters. Therefore, the dynamic parameters can be expected to be more heterogeneous than the kinematic ones and thus also subject to larger uncertainty. Among the dynamic parameters, the characteristic slipweakening distance, $D_{c}$, has the largest relative uncertainty.

Kinematic inversions are known to be nonunique. This is especially well understood when the inversion is formulated in a linear framework, that is, when the slip rate functions along the fault are treated as unknowns (Gallovič et al., 2015; Song \& Dalguer, 2017). In fact, even if the fault geometry and velocity model are set correctly (in synthetic tests), the resulting kinematic models are subject to significant uncertainty due to the vast size of the null space of the inverse problem (Gallovič \& Ampuero, 2015; Gallovič \& Zahradník, 2011; Mai et al., 2016). This limitation is intrinsically due to the incomplete coverage of the wavefield by data recorded only at the surface and to the usable frequency band of the data being limited by attenuation and scattering. The ill-posedness is somewhat reduced if a nonlinear kinematic source inversion approach is adopted, in which a functional form of the slip rates is assumed with a small number of parameters (e.g., rupture time, rise time, and peak slip rate). While the assumption of a slip rate shape works effectively as a constraint, the resulting uncertainty is not negligible. In this context, the dynamic source inversion can be also viewed as a kinematic inversion constrained by the assumed friction law. Compared to the nonlinear kinematic inversion, the dynamic inversion is less restrictive. It allows more general and complex patterns of slip rates and rupture propagation, including phenomena often observed in dynamic rupture models (e.g., Gabriel et al., 2012, 2013; Huang et al., 2014) such as multiple simultaneous fronts (e.g., sub-Rayleigh rupture fronts trailing behind supershear rupture fronts), rupture jumps, renucleating fronts, and backpropagating fronts. Such complexities in the rupture kinematics are physically consistent due to the satisfaction of the prescribed fault friction law. On the other hand, the price to pay is the significant computational burden due to the numerous runs of expensive dynamic rupture simulations.

Peyrat et al. (2004) demonstrated that the dynamic inversion is also nonunique. In particular, trade-offs between strength excess and slip-weakening distance due to the limited frequency band of the data have been previously identified (Guatteri \& Spudich, 2000). Indeed, fracture dynamics theory, in particular the crack tip equation of motion for 2-D problems (Freund, 1990), indicates that the dynamic rupture growth is primarily controlled by stress drop and fracture energy, $G_{c}=D_{c}\left(\tau_{s}-\tau_{d}\right) / 2$ and that the weakening rate $W=\left(\tau_{s}-\tau_{d}\right) / D_{c}$ plays a secondary role. Those concepts suggest that $G_{c}$ would be better resolved than $W$ in a dynamic inversion based on data at frequencies lower than the inverse of the process zone time, which is lower than rupture speed times $W$ divided by shear modulus. To test this idea, we analyze the potential trade-off between slip-weighted $D_{c}$ and strength in our inversion results; see Figure 12. The figure demonstrates a weaker anticorrelation than expected from the simple theoretical argument.

There are many limitations of the present method, some of which may be tackled by more extensive computational power than used here. In particular, we here use a relatively coarse grid parametrization of our dynamic model $(3 \mathrm{~km} \times 2.5 \mathrm{~km})$ and bilinear interpolation to render the parameters to the fine finite difference grid. The coarse parameterization limits the dimension of the model space to be explored by the Monte Carlo sampling. However, it also prevents certain dynamic features. For example, we cannot model an abrupt step of fracture energy $\left(D_{c}\right)$, which would produce an abrupt change of rupture speed that radiates 
a strong omega-squared phase (Madariaga, 1983). Such distinct high-frequency features of the rupture cannot be recognized by the relatively low frequency $(<1 \mathrm{~Hz})$ data used in the inversion.

We have shown that the inverse solution has relatively large uncertainty, which is related to the inherent nonuniqueness of the inverse problem and to the use of a limited frequency range of the data. The results seem to be also severely biased in some aspects. Although to large extent this is mainly due to the diverse parameterizations of the target and inverse models, we attribute it also to (i) insufficient sampling of the posterior and (ii) simplifications considered to speed up the forward dynamic rupture simulations. We discuss these points in detail in the following.

We have dealt with three dynamic parameters being inferred at 171 control points distributed along the fault. Although some of them do not affect the inversion as they are outside of the ruptured area, still the number of visited models versus the number of dimensions is rather low. Hence, further improved convergence would be computationally very expensive. Nevertheless, in future application with improved sampling, the convergence of the sampler could be appropriately assessed, for example, by a Gelman-Rubin test on some of the Markov Chains that sampled the posterior.

The burden of the MC sampling impelled us to limit the computational demand of the dynamic rupture simulations by introducing several simplifying assumptions in the dynamic simulations. In particular, we assumed a vertical fault (although this is partially compensated for by the modification of the velocity model; see section 2.3) and fixed rake orientation. Both are supposedly important when dealing with rupture propagation close to the surface, which is not the case for the Inv1 synthetic model. Nevertheless, such simplifications also give rise to minor biases and a possibility of actually missing some solutions that would be accepted when calculating with a more accurate solver. For these reasons future effort shall aim to develop a rupture simulation code for dynamic inversions with emphasis on its computational efficiency.

Acknowledgments

We thank L. Hanyk and J. Premus for their help with the software development and K. C. Duru for providing us with the WaveQLab3D solver and for his guidance in its usage. We are grateful to the Associate Editor and two anonymous reviewers for their valuable comments that improved the presentation of our results. The paper makes use of synthetic data of Source Inversion Validation benchmark Inv1 (http://equake-rc.info/SIV/sivtools/ benchmarks/). Most of the calculations were carried out on the Salmon supercomputer (Ostrava), supported by the Ministry of Education, Youth and Sports from the Large Infrastructures for Research, Experimental Development and Innovations project IT4Innovations National Supercomputing Center-LM2015070 (IT4I supercomputer). F. G., L. V., and A.-A. G. acknowledge financial support through the bilateral project of the Czech Science Foundation and DFG, 18-06716J and GA 2465/2-1, respectively. J.-P. A. acknowledges support by the French government, through the UCAJEDI Investments in the Future project managed by the National Research Agency (ANR) with the reference number ANR-15-IDEX01. A.-A. G. acknowledges support by the European Union's Horizon 2020 research and innovation program (ExaHyPE, Grant 671698, and ChEESE, Grant 823844), by the Volkswagen Foundation (ASCETE, Grant 88479), and by KAUST-CRG (GAST, Grant ORS-2016-CRG5-3027, and FRAGEN, Grant ORS-2017-CRG6 3389.02).
Let us point out that besides these technical difficulties in real data applications, the bias of the inversion will be even more significant. Indeed, there is rather large uncertainty due to the incomplete knowledge of the velocity model, true fault geometry including segmentation and nonplanarity, fault zone structure, or due to the use of a simplified friction law. The role of the individual simplifications and assumptions needs to be further tested.

Mitigation of the above described technical limitations is a challenge of future applications of the present dynamic inversion method. Nevertheless, the proposed approach provides a venue for earthquake source studies constrained by prescribed friction law. A real data application is demonstrated in our companion paper (Gallovič et al., 2019).

\section{References}

Aki, K., \& Richards, P. G. (2002). Quantitative seismology. Sausalito, California: University Science Books.

Bouchon, M. (1981). A simple method to calculate Green's functions for elastic layered media. Bulletin of the Seismological Society of America, 71, 959-971.

Bouchon, M. (1997). The state of stress on some faults of the San Andreas system as inferred from near-field strong motion data. Journal of Geophysical Research, 102(B6), 11,731-11,744. https://doi.org/10.1029/97JB00623

Brace, W. F., \& Byerlee, J. D. (1966). Stick-slip as a mechanism for earthquakes. Science, 153(3739), 990-992. https://doi.org/10.1126/ science.153.3739.990

Burjánek, J., \& Zahradník, J. (2007). Dynamic stress field of a kinematic earthquake source model with k-squared slip distribution. Geophysical Journal International, 171(3), 1082-1097. https://doi.org/10.1111/j.1365-246X.2007.03548.x

Cirella, A., Piatanesi, A., Cocco, M., Tinti, E., Scognamiglio, L., Michelini, A., et al. (2009). Rupture history of the 2009 L'Aquila (Italy) earthquake from non-linear joint inversion of strong motion and GPS data. Geophysical Research Letters, 36, L19304. https://doi.org/ 10.1029/2009GL039795

Clayton, R., \& Engquist, B. (1977). Absorbing boundary conditions for acoustic and elastic equations. Bulletin of the Seismological Society of America, 67, 1529-1540.

Clévédé, E., Bouin, M.-P., Bukchin, B., Mostinskiy, A., \& Patau, G. (2004). New constraints on the rupture process of the 1999 August 17 Izmit earthquake deduced from estimates of stress glut rate moments. Geophysical Journal International, 159(3), 931-942. https://doi. org/10.1111/j.1365-246X.2004.02304.X

Corish, S. M., Bradley, C. R., \& Olsen, K. B. (2007). Assessment of a nonlinear dynamic rupture inversion technique applied to a synthetic earthquake. Bulletin of the Seismological Society of America, 97(3), 901-914. https://doi.org/10.1785/0120060066

Coutant, O. (1989). Program of numerical simulation AXITRA, Tech. rep., Lab. de Geophys. Interne et Tectonophys., Grenoble, France.

Dalguer, L. A., \& Day, S. M. (2006). Comparison of fault representation methods in finite difference simulations of dynamic rupture. Bulletin of the Seismological Society of America, 96(5), 1764-1778. https://doi.org/10.1785/0120060024

Day, S. M., Dalguer, L. A., Lapusta, N., \& Liu, Y. (2005). Comparison of finite difference and boundary integral solutions to threedimensional spontaneous rupture. Journal of Geophysical Research, 110, B12307. https://doi.org/10.1029/2005JB003813 
Delouis, B., Giardini, D., Lundgren, P., \& Salichon, J. (2002). Joint inversion of InSAR, GPS, teleseismic, and strong-motion data for the spatial and temporal distribution of earthquake slip: Application to the 1999 Izmit mainshock. Bulletin of the Seismological Society of America, 92(1), 278-299. https://doi.org/10.1785/0120000806

Duputel, Z., Jiang, J., Jolivet, R., Simons, M., Rivera, L., Ampuero, J.-P., et al. (2015). The Iquique earthquake sequence of April 2014: Bayesian modeling accounting for prediction uncertainty. Geophysical Research Letters, 42, 7949-7957. https://doi.org/10.1002/ 2015GL065402

Duru, K., \& Dunham, E. M. (2016). Dynamic earthquake rupture simulations on nonplanar faults embedded in 3D geometrically complex, heterogeneous Earth models. Journal of Computational Physics, 305, 185-207. https://doi.org/10.1016/j.jcp.2015.10.021

Falcioni, M., \& Deem, M. W. (1999). A biased Monte Carlo scheme for zeolite structure solution. The Journal of Chemical Physics, 110(3), 1754-1766. https://doi.org/10.1063/1.477812

Fan, W., Shearer, P. M., \& Gerstoft, P. (2014). Kinematic earthquake rupture inversion in the frequency domain. Geophysical Journal International, 199(2), 1138-1160. https://doi.org/10.1093/gji/ggu319

Frankel, A., \& Wennerberg, L. (1989). Rupture process of the $M_{s} 6.6$ Superstition Hills, California, earthquake determined from strongmotion recordings: Application of tomographic source inversion. Bulletin of the Seismological Society of America, 79, 515-541.

Freund, L. (1990). Dynamic fracture mechanics, Cambridge monographs on mechanics. Cambridge: Cambridge University Press. https://doi. org/10.1017/CBO9780511546761

Fukuyama, E., \& Mikumo, T. (1993). Dynamic rupture analysis: Inversion for the source process of the 1990 Izu-Oshima, Japan, earthquake. Journal of Geophysical Research, 98(B4), 6529-6542. https://doi.org/10.1029/92JB02451

Gabriel, A.-A., Ampuero, J. P., Dalguer, L. A., \& Mai, P. M. (2012). The transition of dynamic rupture styles in elastic media under velocityweakening friction. Journal of Geophysical Research, 117, B09311. https://doi.org/10.1029/2012JB009468

Gabriel, A.-A., Ampuero, J.-P., Dalguer, L. A., \& Mai, P. M. (2013). Source properties of dynamic rupture pulses with off-fault plasticity. Journal of Geophysical Research: Solid Earth, 118, 4117-4126. https://doi.org/10.1002/jgrb.50213

Gallovič, F., \& Ampuero, J.-P. (2015). A new strategy to compare inverted rupturemodels exploiting the eigen-structure of the inverse problem. Seismological Research Letters, 86, 1679-1689.

Gallovič, F., Imperatori, W., \& Mai, P. M. (2015). Effects of three-dimensional crustal structure and smoothing constraint on earthquake slip inversions: Case study of the Mw6.3 2009 L'Aquila earthquake. Journal of Geophysical Research: Solid Earth, 120, 428-449. https:// doi.org/10.1002/2014JB011650

Gallovič, F., Valentová, L'., Ampuero, J.-P., \& Gabriel, A.-A. (2019). Bayesian dynamic finite-fault inversion: 2. Application to the 2016 Mw6.2 Amatrice, Italy, earthquake. Journal of Geophysical Research: Solid Earth, 124, 6970-6988. https://doi.org/10.1029/2019JB017512

Gallovič, F., \& Zahradník, J. (2011). Toward understanding slip-inversion uncertainty and artifacts: 2. Singular value analysis. Journal of Geophysical Research, 116, B02309. https://doi.org/10.1029/2010JB007814

Gallovič, F., Zahradník, J., Křížová, D., Plicka, V., Sokos, E., Serpetsidaki, A., \& Tselentis, G.-A. (2009). From earthquake centroid to spatial-temporal rupture evolution: $M_{w} 6.3$ Movri Mountain earthquake, June 8, 2008, Greece. Geophysical Research Letters, 36, L21310. https://doi.org/10.1029/2009GL040283

Goto, H., Yamamoto, Y., \& Kita, S. (2012). Dynamic rupture simulation of the 2011 off the Pacific coast of Tohoku earthquake: Multi-event generation within dozens of seconds. Earth, Planets and Space, 64(12), 1167-1175. https://doi.org/10.5047/eps.2012.06.002

Graves, R. W. (1996). Simulating seismic wave propagation in 3D elastic media using staggered-grid finite differences. Bulletin of the Seismological Society of America, 86, 1091-1106.

Guatteri, M., \& Spudich, P. (2000). What can strong-motion data tell us about slip-weakening fault-friction laws? Bulletin of the Seismological Society of America, 90(1), 98-116. https://doi.org/10.1785/0119990053

Guatteri, M., Spudich, P., \& Beroza, G. C. (2001). Inferring rate and state friction parameters from a rupture model of the 1995 Hyogo-ken Nanbu (Kobe) Japan earthquake. Journal of Geophysical Research, 106(B11), 26,511-26,521. https://doi.org/10.1029/ 2001JB000294

Hallo, M., \& Gallovič, F. (2016). Fast and cheap approximation of Green functions uncertainty for waveform-based earthquake source inversions. Geophysical Journal International, 207(2), 1012-1029. https://doi.org/10.1093/gji/ggw320

Hallo, M., Opršal, I., Asano, K., \& Gallovič, F. (2019). Seismotectonics of the 2018 Northern Osaka M6.1 earthquake and its aftershocks: Joint movements on strike-slip and reverse faults in inland Japan. Earth, Planets and Space, 71(1), 34. https://doi.org/10.1186/s40623019-1016-8

Harris, R. A., Barall, M., Aagaard, B., Ma, S., Roten, D., Olsen, K., et al. (2018). A suite of exercises for verifying dynamic earthquake rupture codes. Seismological Research Letters, 89(3), 1146-1162. https://doi.org/10.1785/0220170222

Hartzell, S., Liu, P., Mendoza, C., Ji, C., \& Larson, K. M. (2007). Stability and uncertainty of finite-fault slip inversions: Application to the 2004 Parkfield, California, earthquake. Bulletin of the Seismological Society of America, 97(6), 1911-1934. https://doi.org/10.1785/ 0120070080

Hartzell, S. H., \& Heaton, T. H. (1983). Inversion of strong ground motion and teleseismic waveform data for the fault rupture history of the 1979 Imperial Valley, California, earthquake. Bulletin of the Seismological Society of America, 73, 1553-1583.

Huang, Y., Ampuero, J. P., \& Helmberger, D. V. (2014). Earthquake ruptures modulated by waves in damaged fault zones. Journal of Geophysical Research: Solid Earth, 119, 3133-3154. https://doi.org/10.1002/2013JB010724

Ide, S., \& Takeo, M. (1996). The dynamic rupture process of the 1993 Kushiro-oki earthquake. Journal of Geophysical Research, 101(B3), 5661-5675. https://doi.org/10.1029/95JB00959

Ji, C., Wald, D., \& Helmberger, D. V. (2002). Source description of the 1999 Hector Mine, California, earthquake. Part I: Wavelet domain inversion theory and resolution analysis. Bulletin of the Seismological Society of America, 92(4), 1192-1207. https://doi.org/10.1785/ 0120000916

Kennett, B. L. N., \& Kerry, N. J. (1979). Seismic waves in a stratified half space. Geophysical Journal International, 57(3), 557-583. https:// doi.org/10.1111/j.1365-246X.1979.tb06779.x

Kristek, J., Moczo, P., \& Archuleta, R. J. (2002). Efficient methods to simulate planar free surface in the 3D 4th-order staggered-grid finitedifference schemes. Studia Geophysica et Geodaetica, 46(2), 355-381. https://doi.org/10.1023/A:1019866422821

Levander, A. (1988). Fourth-order finite-difference P-SV seismograms. Geophysics, 53(11), 1425-1436. https://doi.org/10.1190/1.1442422

Madariaga, R. (1983). High frequency radiation from dynamic earthquake. Annales de Geophysique, 1, 17-23.

Madariaga, R. (2005). Boundary conditions for numerical modeling of seismic ruptures, EGU annual meeting, Vienna. http://www.geologie.ens.fr/ madariag/Programs/egu2005b

Madariaga, R., Olsen, K. B., \& Archuleta, R. J. (1998). Modelling dynamic rupture in a 3D earthquake fault model. Bulletin of the Seismological Society of America, 88(5), 1182-1197. 
Madariaga, R., \& Ruiz, S. (2016). Earthquake dynamics on circular faults: A review 1970-2015. Journal of Seismology, 20(4), $1235-1252$. https://doi.org/10.1007/s10950-016-9590-8

Mai, P. M., Schorlemmer, D., Page, M., Ampuero, J.-P., Asano, K., Causse, M., et al. (2016). The earthquake-source inversion validation (SIV) project. Seismological Research Letters, 87(3), 690-708. https://doi.org/10.1785/0220150231

Metropolis, N., Rosenbluth, A., Rosenbluth, M., Teller, A., \& Teller, E. (1953). Equations of state calculations by fast computing machines. The Journal of Chemical Physics, 21(6), 1087-1092. https://doi.org/10.1063/1.1699114

Monelli, D., \& Mai, M. P. (2008). Bayesian inference of kinematic earthquake rupture parameters through fitting of strong motion data. Geophysical Journal International, 173(1), 220-232. https://doi.org/10.1111/j.1365-246X.2008.03733.x

Niemeijer, A., Marone, C., \& Elsworth, D. (2010). Fabric induced weakness of tectonic faults. Geophysical Research Letters, 37, L03304. https://doi.org/10.1029/2009GL041689

Ohnaka, M. (2013). The physics of rock failure and earthquakes. Cambridge: Cambridge University Press. https://doi.org/10.1017/ CBO9781139342865

Okada, Y. (1992). Internal deformation due to shear and tensile faults in a half-space. Bulletin of the Seismological Society of America, 82, $1018-1040$.

Olsen, K. B., Madariaga, R., \& Archuleta, R. J. (1997). Three-dimensional dynamic simulation of the 1992 Landers earthquake. Science, 278, 834-838.

Olson, A. H., \& Anderson, J. G. (1988). Implications of frequency-domain inversion of earthquake ground motions for resolving the spacetime dependence of slip on an extended fault. Geophysical Journal International, 94(3), 443-455. https://doi.org/10.1111/j.1365246X.1988.tb02267.x

Peyrat, S., \& Olsen, K. B. (2004). Nonlinear dynamic rupture inversion of the 2000 Western Tottori, Japan, earthquake. Geophysical Research Letters, 31, L05604. https://doi.org/10.1029/2003GL019058

Peyrat, S., Olsen, K. B., \& Madariaga, R. (2001). Dynamic modeling of the 1992 Landers earthquake. Journal of Geophysical Research, 106(B11), 26,467-26,482.

Peyrat, S., Olsen, K. B., \& Madariaga, R. (2004). Which dynamic rupture parameters can be estimated from strong ground motion and geodetic data? Pure and Applied Geophysics, 161, 2155-2169.

Piatanesi, A., Cirella, A., Spudich, P., \& Cocco, M. (2007). A global search inversion for earthquake kinematic rupture history: Application to the 2000 western Tottori, Japan earthquake. Journal of Geophysical Research, 112, B07314. https://doi.org/10.1029/2006JB004821

Pizzi, A., di Domenica, A., Gallovič, F., Luzi, L., \& Puglia, R. (2017). Fault segmentation as constraint to the occurrence of the main shocks of the 2016 Central Italy seismic sequence. Tectonics, 36, 2370-2387. https://doi.org/10.1002/2017TC004652

Ragon, T., Sladen, A., \& Simons, M. (2018). Accounting for uncertain fault geometry in earthquake source inversions-I: Theory and simplified application. Geophysical Journal International, 214(2), 1174-1190. https://doi.org/10.1093/gji/ggy187

Ragon, T., Sladen, A., \& Simons, M. (2019). Accounting for uncertain fault geometry in earthquake source inversions-II: Application to the $M_{w} 6.2$ Amatrice earthquake, central Italy. Geophysical Journal International, 218(1), 689-707. https://doi.org/10.1093/gji/ggz180

Ruina, A. L. (1983). Slip instability and state variable friction laws. Journal of Geophysical Research, 88(B12), 10359-10370. https://doi.org/ 10.1029/JB088iB12p10359

Ruiz, S., \& Madariaga, R. (2011). Determination of the friction law parameters of the $M_{w} 6.7$ Michilla earthquake in northern Chile by dynamic inversion. Geophysical Research Letters, 38, L09317. https://doi.org/10.1029/2011GL047147

Ruiz, S., \& Madariaga, R. (2013). Kinematic and dynamic inversion of the 2008 Northern Iwate earthquake. Bulletin of the Seismological Society of America, 103(2A), 694-708. https://doi.org/10.1785/0120120056

Sambridge, M. (2013). A Parallel Tempering algorithm for probabilistic sampling and multimodal optimization. Geophysical Journal International, 196, 357-374.

Sambridge, M., \& Mosegaard, K. (2002). Monte Carlo methods in geophysical inverse problems. Reviews of Geophysics, 40(3), 1009. https:// doi.org/10.1029/2000RG000089

Sekiguchi, H., Irikura, K., \& Iwata, T. (2002). Source inversion for estimating the continuous slip distribution on a fault-Introduction of Green's functions convolved with a correction function to give moving dislocation effects in subfaults. Geophysical Journal International, 150(2), 377-391. https://doi.org/10.1046/j.1365-246X.2002.01669.x

Shao, G., \& Ji, C. (2012). What the exercise of the SPICE source inversion validation BlindTest 1 did not tell you. Geophysical Journal International, 189(1), 569-590. https://doi.org/10.1111/j.1365-246X.2012.05359.x

Sokos, E., \& Zahradník, J. (2013). Evaluating centroid-moment-tensor uncertainty in the new version of ISOLA software. Seismological Research Letters, 84(4), 656-665. https://doi.org/10.1785/0220130002

Sokos, E., Zahradník, J., Gallovič, F., Serpetsidaki, A., Plicka, V., \& Kiratzi, A. (2016). Asperity break after 12 years: The $M_{w} 6.42015$ Lefkada (Greece) earthquake. Geophysical Research Letters, 43, 6137-6145. https://doi.org/10.1002/2016GL069427

Song, S. G., \& Dalguer, L. A. (2017). Synthetic source inversion tests with the full complexity of earthquake source processes, including both supershear rupture and slip reactivation. Pure and Applied Geophysics, 174(9), 3393-3418. https://doi.org/10.1007/ s00024-017-1514-1

Spudich, P., \& Archuleta, R. J. (1987). Techniques for earthquake ground motion calculation with applications to source parameterization of finite faults. In B. A. Bolt (Ed.), Seismic strong motion synthetics (pp. 205-265). San Diego, CA: Acad. Press. https://doi.org/10.1016/ B978-0-12-112251-5.50009-1

Tanırcan, G., Dalguer, L., Bekler, F. N., \& Meral Özel, N. (2017). Dynamic upture modelling of the 1999 Düzce, Turkey earthquake. Pure and Applied Geophysics, 174(9), 3343-3355. https://doi.org/10.1007/s00024-017-1531-0

Tarantola, A. (2005). Inverse problem theory and methods for model parameter estimation. SIAM. https://doi.org/10.1137/1.9780898717921

Tarantola, A., \& Valette, B. (1982). Generalized nonlinear inverse problems solved using the least squares criterion. Reviews of Geophysics, 20(2), 219-232. https://doi.org/10.1029/RG0201002p00219

Tinti, E., Cocco, M., Fukuyama, E., \& Piatanesi, A. (2009). Dependence of slip weakening distance $\left(D_{c}\right)$ on final slip during dynamic rupture of earthquakes. Geophysical Journal International, 177(3), 1205-1220. https://doi.org/10.1111/j.1365-246X.2009.04143.x

Twardzik, C., Das, S., \& Madariaga, R. (2014). Inversion for the physical parameters that control the source dynamics of the 2004 Parkfield earthquake. Journal of Geophysical Research: Solid Earth, 119, 7010-7027. https://doi.org/10.1002/2014JB011238

Ulrich, T., Gabriel, A. A., Ampuero, J. P., \& Xu, W. (2019). Dynamic viability of the $2016 M_{w} 7.8$ Kaikoura earthquake cascade on weak crustal faults. Nature Communications, 10(1), 1213. https://doi.org/10.1038/s41467-019-09125-w

Uphoff, C., Rettenberger, S., Bader, M., Madden, E. H., Ulrich, T., Wollherr, S., \& Gabriel, A.-A. (2017). Extreme scale multi-physics simulations of the tsunamigenic 2004 Sumatra megathrust earthquake, in Proceedings of the International Conference for High Performance Computing, Networking, Storage and Analysis (pp. 1-16). Denver, CO: ACM. https://doi.org/10.1145/3126908.3126948 
Viesca, R. C., \& Garagash, D. I. (2015). Ubiquitous weakening of faults due to thermal pressurization. Nature Geoscience, 8(11), 875-879. https://doi.org/10.1038/ngeo2554

Weng, H., \& Yang, H. (2018). Constraining frictional properties on fault by dynamic rupture simulations and near-field observations. Journal of Geophysical Research: Solid Earth, 123, 6658-6670. https://doi.org/10.1029/2017JB015414

Yagi, Y., \& Fukahata, Y. (2011). Introduction of uncertainty of Green's function into waveform inversion for seismic source processes Geophysical Journal International, 186(2), 711-720. https://doi.org/10.1111/j.1365-246X.2011.05043.x

Zahradník, J., \& Gallovič, F. (2010). Toward understanding slip inversion uncertainty and artifacts. Journal of Geophysical Research, 115, B09310. https://doi.org/10.1029/2010JB007414

Zhang, W., Iwata, T., Irikura, K., Sekiguchi, H., \& Bouchon, M. (2003). Heterogeneous distribution of the dynamic source parameters of the 1999 Chi-Chi, Taiwan, earthquake. Journal of Geophysical Research, 108(B5), 2232. https://doi.org/10.1029/2002JB001889 